\title{
REVIEW ARTICLE OPEN \\ Immune checkpoint molecules in natural killer cells as potential targets for cancer immunotherapy
}

\author{
Yuqing Cao ${ }^{1}$, Xiaoyu Wang ${ }^{2}$, Tianqiang $\operatorname{Jin}^{1}$, Yu Tian ${ }^{1}$, Chaoliu Dai ${ }^{1}$, Crystal Widarma ${ }^{3}$, Rui Song ${ }^{3}$ and Feng Xu ${ }^{1}$
}

Recent studies have demonstrated the potential of natural killer (NK) cells in immunotherapy to treat multiple types of cancer. NK cells are innate lymphoid cells that play essential roles in tumor surveillance and control that efficiently kill the tumor and do not require the major histocompatibility complex. The discovery of the NK's potential as a promising therapeutic target for cancer is a relief to oncologists as they face the challenge of increased chemo-resistant cancers. NK cells show great potential against solid and hematologic tumors and have progressively shown promise as a therapeutic target for cancer immunotherapy. The effector role of these cells is reliant on the balance of inhibitory and activating signals. Understanding the role of various immune checkpoint molecules in the exhaustion and impairment of NK cells when their inhibitory receptors are excessively expressed is particularly important in cancer immunotherapy studies and clinical implementation. Emerging immune checkpoint receptors and molecules have been found to mediate NK cell dysfunction in the tumor microenvironment; this has brought up the need to explore further additional NK cell-related immune checkpoints that may be exploited to enhance the immune response to refractory cancers. Accordingly, this review will focus on the recent findings concerning the roles of immune checkpoint molecules and receptors in the regulation of NK cell function, as well as their potential application in tumor immunotherapy.

Signal Transduction and Targeted Therapy (2020)5:250

; https://doi.org/10.1038/s41392-020-00348-8

\section{INTRODUCTION}

Natural killer (NK) cells are cytotoxic lymphocytes in the family of innate lymphoid cells that play essential roles in the first line of defense against cancer and viral infections. ${ }^{1-3}$ Human NK cells make up to 15 percent of the circulating lymphocytes and are associated with the killing and destruction of microbially infected and malignantly autologous and allogenic cells. ${ }^{4}$

NK cells have been determined to demonstrate antitumor cell cytotoxicity in the absence of prior sensitization and the subsequent production of cytokines alongside chemokines that have an effect on regulating certain immune responses. ${ }^{4}$ Some types of cancer, such as invasive breast carcinoma, bladder urothelial carcinoma, renal clear cell carcinoma, colon adenocarcinoma, lung squamous cell carcinoma, lower-grade glioma, pancreatic adenocarcinoma, stomach adenocarcinoma, cutaneous melanoma, uterine corpus endometrial carcinoma, and thyroid carcinoma, are associated with a deficiency of the number of NK cells leading to poor clinical outcomes. ${ }^{5}$ Many cancer types have demonstrated an imbalance of immune-regulated signals in NK cells. Inhibitory and activating receptors are expressed on the surface of NK cells and contribute to the execution of various NK cell functions. ${ }^{4}$ NK cells express the non-HLA-class I-specific inhibitory receptors (such as programmed cell death protein 1 $[\mathrm{PD}-1]{ }^{6,7} \mathrm{~T}$ cell immunoreceptor with $\mathrm{lg}$ and immunoreceptor tyrosine-based inhibition motif [ITIM] ${ }^{8}$ domains [TIGIT], ${ }^{9,10}$ a cluster of differentiation 112 receptor [CD112R], CD96, interleukin-1 receptor 8 [IL-1R8], and $\mathrm{T}$ cell immunoglobulin and mucindomain-containing molecule 3 [TIM-3]), as well as activating receptors (such as NK group 2 [NKG2] family of receptor D [NKG2D] and coreceptor [CD226]). ${ }^{4,11-13}$ NK cells also express the HLA-class I-specific inhibitory receptors (such as killer cell immunoglobin-like receptor (KIR), NKG2A, and lymphocyte activation gene-3 [LAG-3]). ${ }^{14}$ Although more researches have shown that the blockade of checkpoint inhibitory receptors may be crucial in effectively reversing $T$ cell exhaustion and the restoration of the antitumor capacity of $\mathrm{T}$ cells, ${ }^{15}$ there has been growing interest in the immune effects these treatments may have on NK cells as well.

Immune checkpoint inhibitors (ICls) represent some of the most efficient immunotherapeutic approaches currently used to treat cancer. ${ }^{16} \mathrm{ICls}$ are antibodies that serve the role of binding to inhibitory molecules on the tumor-infiltrating surfaces of lymphocytes and thereby allowing the reactivation of antitumor immune responses. ${ }^{17}$ One of the promising discoveries to enhance antitumor activity and survival is the dual targeting of chimeric antigen-based receptor-engineered NK cells of glioblastoma, which has been found to overcome the heterogeneity of specific expression of target antigens and enhances the antitumor activity and survival. ${ }^{17,18}$ Moreover, immune checkpoint receptors (IC) contribute either positively or negatively in the regulation of activating the host immune response, preventing unwanted reactions that may affect healthy tissues. ${ }^{19}$

The term IC is mainly used about the inhibitory ICs critical in controlling $\mathrm{NK}$ and cytotoxic $\mathrm{CD}^{+}{ }^{+} \mathrm{T}$ cells because of its high cytotoxic potential. ${ }^{19}$ Therefore, specific ICs are described concerning their regulation of either NK cell or T cell activity. ${ }^{19}$

\footnotetext{
${ }^{1}$ Department of General Surgery, Shengjing Hospital of China Medical University, 110004 Shenyang, China; ${ }^{2}$ College of Life and Health Science, Northeastern University, 110819

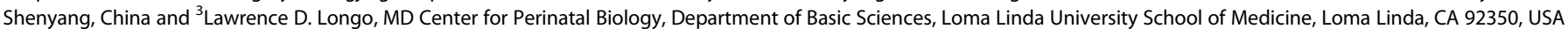
Correspondence: Rui Song (rsong01@llu.edu) or Feng Xu (xuf@sj-hospital.org)

These authors contributed equally: Yuqing Cao, Xiaoyu Wang
}

Received: 19 May 2020 Revised: 13 September 2020 Accepted: 21 September 2020

Published online: 29 October 2020 


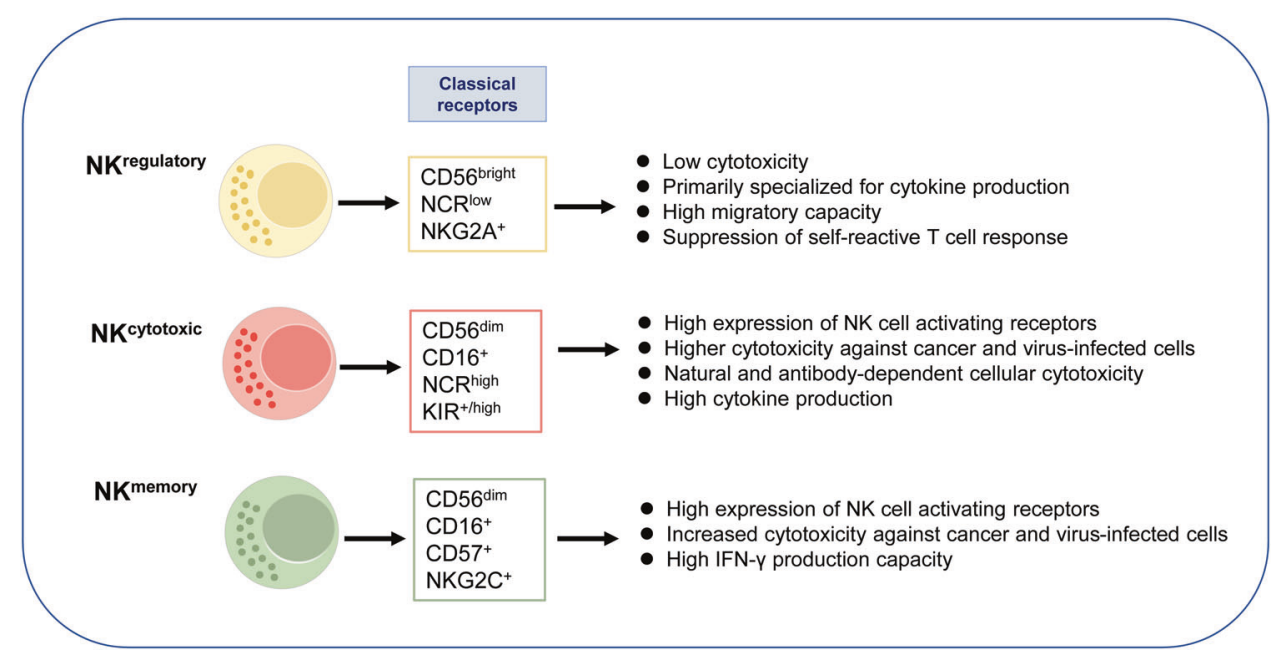

Fig. 1 Phenotypical and functional properties of NK cells. According to classical receptors (for example, adhesion molecules-CD56 and CD57, activating receptors-CD16, NCR, KIR and NKG2C, inhibitory receptors-NKG2A), NK cells are subdivided into regulatory and cytotoxic phenotypes and memory NK cells. Therefore, these subsets of NK cells appear to be functionally different

NK cell activation is mainly regulated by receptors from the natural cytotoxicity receptor, NKG2, and KIR families. ${ }^{19-22}$ For example, activating and inhibitory KIR receptors serve to control the development and function of NK cell immunity while adjusting to the tumor microenvironment (TME). ${ }^{23,24}$ The interactions between KIRs and the corresponding HLA class I ligands contribute to the mediation of NK cell self-tolerance or cytotoxicity against the transformed cells. ${ }^{24,25}$ The control of unwanted damage of healthy cells by the NK cells is regulated by HLAclass I-specific receptors, which contain a fail-safe mechanism. Recent studies show that NKG2A forms a crucial checkpoint for controlling $\mathrm{T}$ cell and NK cell activation in cancerous conditions. ${ }^{24,26}$ The checkpoint receptors mediate the delivery of multiple signals, the balance of which determines if NK cells kill their target cells, such as stressed cells and tumor cells, or remain inactive. ${ }^{27-29}$

Dysregulation of checkpoint receptor signaling contributes to NK cell dysfunction. ${ }^{30}$ Furthermore, the downregulation of activating NK cell receptors and the upregulation of inhibitory receptors have been reported in multiple tumors. For example, NK cells expressed decreased levels of CD226 in various types of cancers, including myeloma, breast cancer, and myeloid leukemia. ${ }^{14,15,31}$ Notably, the downregulation of activating NK cell receptors, sometimes by helix-loop-helix protein ID2, serves as an active suppression and can be restored in the TME, such as by the repression of GSK3.$^{14,32,33}$ As highlighted earlier, ICs can further be described as being membrane molecules that are mainly located, although not exclusively, on T lymphocytes and NK cells that act after recognizing appropriate ligands on the antigen-presenting cells (APC) or the target cells. Therefore, ICs can play either a negative or positive role in the processes of lymphocyte activation. The detrimental regulation of NK cell function also involves the upregulation of inhibitory receptors. For example, PD-1 upregulation was recently found in NK cells from multiple myeloma, Kaposi sarcoma, and head and neck cancer patients. ${ }^{16,34-36}$ TIGIT expression on NK cells was further upregulated in tumor regions compared with peritumoral regions in colorectal tumors. ${ }^{10,24,37-39}$ CD96, CD112R, TIM-3, NKG2A, LAG-3, and IL-1R8 are other significant receptors that have been proved to be upregulated on NK cells in cancers. ${ }^{26,40,41}$

Aside from this, the expression of various ligands on cancer cells has been reported to target common signaling molecules. The targeted interaction modifies the function of NK cells by regulating its numerous inhibitory and activating receptors. For instance, transformed cells may lose or decrease their HLA-I expression during cancer progression due to the activation of inhibitory NK cell receptors bound to their surfaces. ${ }^{26}$ Increased activation of inhibitory receptors and decreased stimulation of activating receptors reduce the antitumor response in the NK cells, and facilitate the induction of tumor immune escape. Several solid tumor types create an immunosuppressive microenvironment that prevents T cell or NK cell-mediated lysis through inhibitory ligands, such as the PD-L1 expressed on the surfaces of the cancer cells. ${ }^{6,42-45}$ The modulation of these checkpoint molecules and the development of inhibitory receptor blockade provide a vital strategy to improve NK cell function, such as by Diacylglycerol Kinase zeta. ${ }^{46-49}$

NK cells have essential roles in tumor surveillance and control. Some studies have identified the presence of dysfunctional NK cells in some cancer patients. ${ }^{4,14,16,50}$ Due to the inability to preserve their primary role, the dysfunctional NK cells allow certain cancers to escape the immune surveillance. ${ }^{17,51,52}$ Thus, restoring the function of NK cells in cancer patients may be a promising approach for immunotherapy. ${ }^{53,54}$ In the following sections, a detailed analysis of NK cell immune checkpoint receptors and their ligands will be discussed along with their prospective influence in cancer immunotherapy. ${ }^{55-57}$

\section{NK CELL PHENOTYPE AND FUNCTION}

NK cells display a range of phenotypic and functional characteristics (Fig. 1). The activated phenotype of NK cells is directly related to the progression of a disease. For example, there are phenotypic and functional differences that NK cells exhibit in patients with the immune reconstitution inflammatory syndrome compared with healthy individuals. ${ }^{58}$ The phenotype and cytotoxic function of NK cells are closely associated. Based on the experiment conducted to explore the extent to which NK cells in patients with cancers can be activated to kill cancer cells when stimulated by cytokines, NK cells have natural cytotoxicity that destroys cancer cells in cancer patients. ${ }^{59}$

On the other hand, cytokine-mediated cytotoxicity is regulated by a balance between the inhibitory and activating receptors. NK cells' natural cytotoxicity requires co-engagement of several activating receptors such as KIR2DSs. ${ }^{60}$ Stimulated NK cells, such as tumor-primed cells, are phenotypically different from resting NK cells and characteristic with upregulation of NK cells' expression of the inhibitory receptors and/or downregulation of the activating receptors. ${ }^{59}$ 


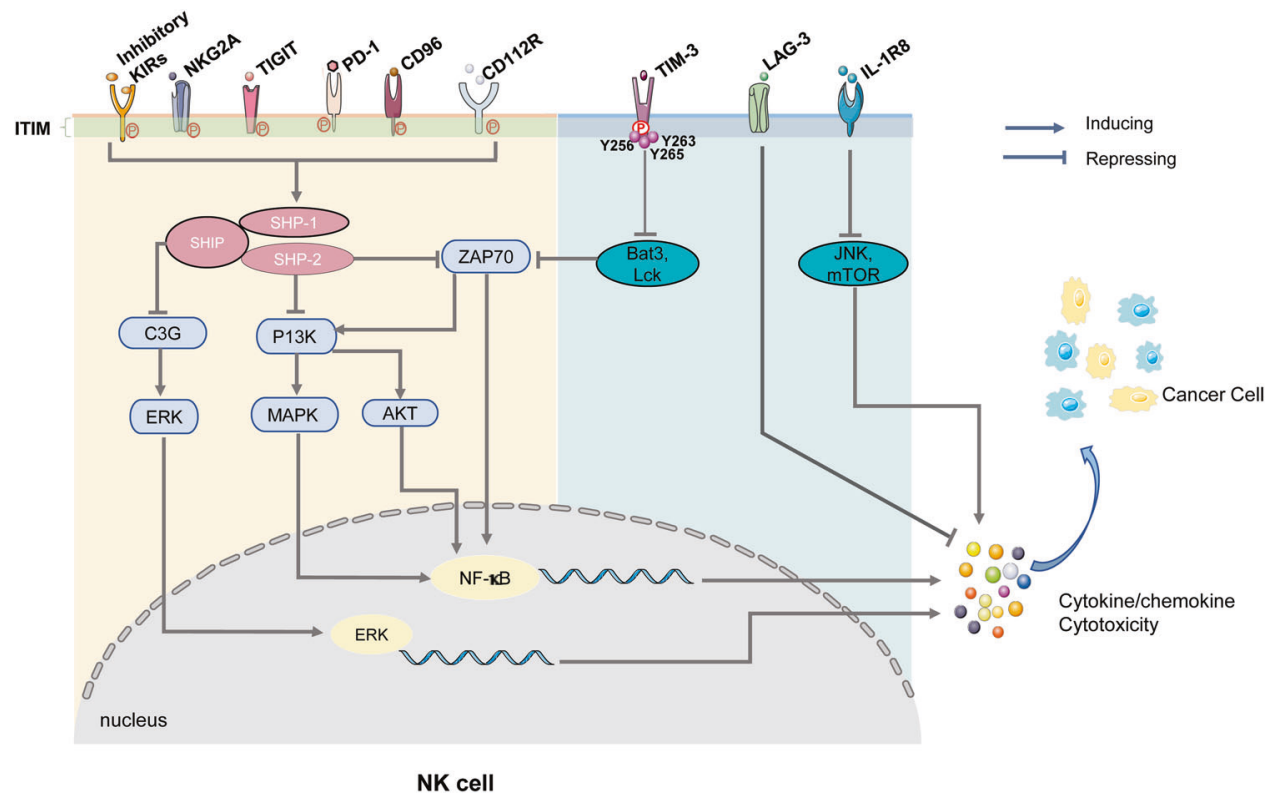

Fig. 2 NK cell inhibitory receptor signaling is required for immune escape. Augmentation of inhibitory checkpoint NK cell receptor activation reduces the antitumor response in NK cells and facilitates the induction of tumor immune escape. In the tumor microenvironment, most of these receptors' stimulation results in the phosphorylation of immunoreceptor tyrosine tail (ITT)-like motifs on their intracytoplasmic tails. After that, these immunoreceptor tyrosine-based inhibition motifs (ITIM) recruit phosphatases (such as Src homology domain-containing tyrosine phosphatase [SHP] and SH2 domain-containing inositol-5-phosphatase [SHIP]) that inhibit downstream signaling and ultimately suppress NK cell cytotoxic activity. Further research is necessary to understand better the signal mechanisms of these checkpoint receptors (including CD96, CD112R, and LAG-3). Nonetheless, studies have shown that these inhibitory signals attenuate NK cell cytotoxicity and antitumor cytokine/chemokine release, allowing tumor immune escape

\section{CHECKPOINT RECEPTORS AND LIGANDS IN NK CELL DYSFUNCTION}

As previously mentioned, NK cell function is regulated and maintained by the balance of inhibitory and activating receptors responsible for the preservation of the cell's steady internal environment (cell homeostasis). In cancer microenvironments, the function of antitumor NK cells is affected by the decreasing expression of activating receptors and their ligands, as well as the increased expression of inhibitory immune checkpoint molecules; this facilitates the tumor immune escape (Figs. 2 and 3) and allows for the development of various cancer types. ${ }^{61-64}$ NK cell activation is primarily controlled by the dynamic balance between inhibitory and activating receptor signaling. ${ }^{65}$ As an example, NK cells express cell surface inhibitory receptors antagonizing to the activation pathways through protein tyrosine phosphatases. ${ }^{66}$ The nature of the typical inhibitory receptor, KIR, contributes to the recognition of self-antigens and subsequently provides negative signals that lead to the eventual suppression of NK cell activation through inhibition of the stimulatory signaling pathway. The ligand of the KIR is the major histocompatibility complex (MHC) class I molecule and is present in healthy cells. The inhibitory cell surface receptors are innately characterized by ITIM present in the cytoplasmic domains of various inhibitory receptors. $^{66}$

Killer cell immunoglobulin-like receptor (KIR) family

As highlighted earlier, NK cells across development express inhibitory KIR receptors that are specific for MHC class I antigens in a variegated and stochastic manner. ${ }^{21,67} \mathrm{KIRs}$ are divided into haplotypes $A$ and $B$, where $A$ is more frequent and mainly constituted of inhibitory receptors, while $B$ contains both types of receptors with a predominance of the activating type. ${ }^{67}$ The repertoire of NK cell KIR is highly dependent on both HLA and KIR polymorphisms. ${ }^{21}$ The inhibitory KIRs inhibit NK cell activity through ITIM-based recruitment of protein tyrosine phosphatases (SHP-1 and SHP-2) responsible for dephosphorylation of the surrounding tyrosine kinases and the adapter proteins, which include DAP-10. ${ }^{67}$ As a diversified and polymorphic group of NK cell receptors that comprise inhibitory and activating KIRs, each member of the KIR family recognizes a particular HLA class I allotype from $-A,-B,-C$ as the ligand. ${ }^{26}$ As an example, the inhibitory KIR2DL1, KIR2DL2, and KIR2DL3 affiliate with HLA-C as their ligand, whereas HLA-A and HLA-B serve as the ligands attached to other KIRs that include the inhibitory KIR3DL2 and KIR3DL3. ${ }^{68,69}$ Furthermore, the KIRs, ligands for KIRs, and MHC-I molecules exhibit high natural polymorphism. ${ }^{26}$

PD-1

In the context of cancer immunology, co-inhibitory signaling molecules are well described for T cells. One of the most notable is PD-1, also known as CD279. This receptor also occurs in NK cells and has been shown to reduce NK cell response. ${ }^{70}$ PD-1 is highly expressed on CD56 $6^{\text {dim }}$ NK cells in the peripheral blood of approximately one-quarter of healthy humans. ${ }^{71}$ In ovarian carcinoma, the CD56 dim NK cells with high PD- 1 expression have been found to have the phenotypic characteristics of fully mature NK cells with the NKG2 $\mathrm{A}^{-} \mathrm{KIR}^{+} \mathrm{CD} 57^{+}$phenotype and are increased in patients. ${ }^{72}$ A recent study reported PD-1 expression by NK cells in the malignant pleural effusions of patients with primary mesothelioma or metastatic tumors, including lung cancer, intestinal adenocarcinoma, uterine cancer, breast cancer, and bladder carcinoma. ${ }^{73,74}$ Another recent study warned of the possibility of PD-1 leading promoting hyperprogression of cancer if amplified by PD-1 blockade, ${ }^{36}$ which can be the mechanism of how the receptor downregulates NK cell response. ${ }^{75,76}$

PD-1 binds to the ligands PD-L1 and PD-L2 to mediate inactivation of immune cells, including NK cells. ${ }^{44,77,78}$ Studies showed that PD-1/PD-L1 interaction in NK cells was required in $\mathrm{MHC}^{+}$and $\mathrm{MHC}^{-}$tumors. In mouse lymphoma tumor models, PD1 inhibits NK cell-dependent immune surveillance and facilitates the escape of tumor cells from the NK cell response. ${ }^{79-81}$ More so, PD-1 contains an extracellular variable immunoglobulin ( $\operatorname{lgV}$ ) 


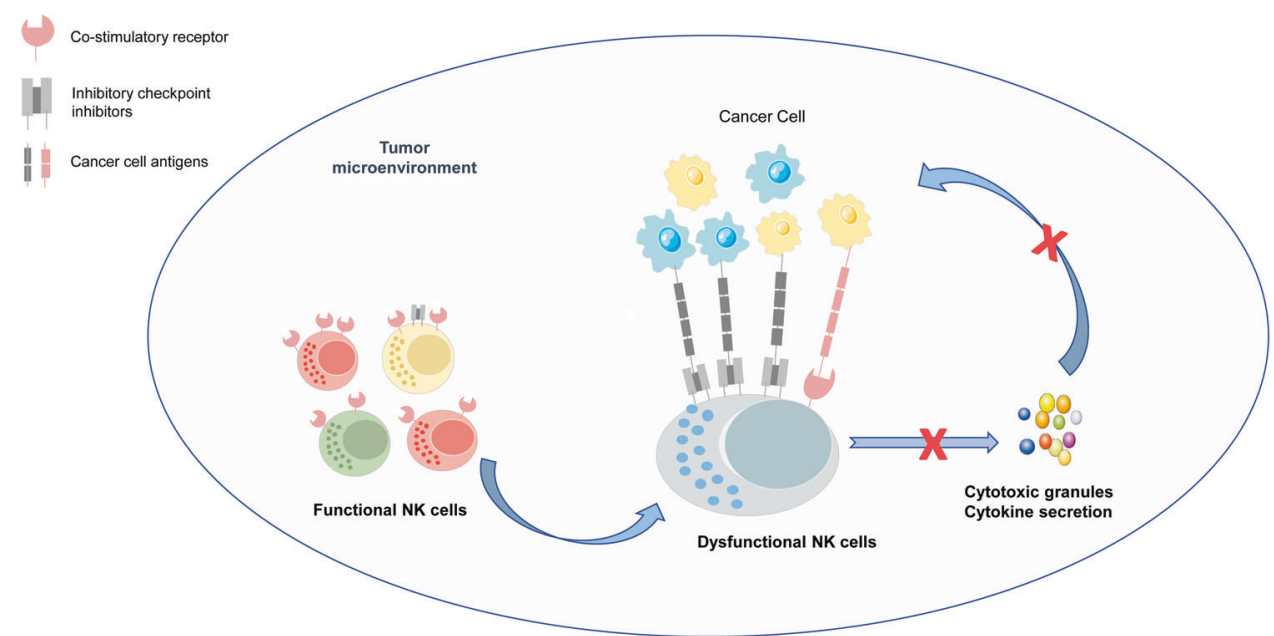

Fig. 3 Schematic representation of a model of NK cell immune checkpoint receptors in tumor cell recognition. NK cell function is regulated and maintained by the balance of inhibitory and activating receptor signals. In the tumor microenvironment, NK cells transform to become dysfunctional cells with enhanced expression of inhibitory immune checkpoints and decreased expression of activating immune checkpoints. This imbalance of signaling transmission induces NK cell dysfunction and the extinction of antitumor immune response. Blockade of these inhibitory checkpoint molecules with the use of immune receptor inhibitors (ICI) (e.g., anti-KIR (lirilumab), anti-PD-1 (sintilimab and pembrolizumab), anti-NKG2A (monalizumab)), as well as activation of co-stimulatory receptor signaling by inducing co-stimulatory receptor expression (e.g., NKG2D ligand $\alpha 3$ domain-specific antibodies, cytokines (IL-2, IL-15, and IL-21), demethylating agents, histamine, anti-TGF- $\beta$ monoclonal antibodies, TGF- $\beta$ receptor inhibitors, TGF- $\beta$ antisense oligonucleotides), can restore the antitumor activity of NK cells

domain, a transmembrane region, and an intracellular tail with two phosphorylation sites; one phosphorylation site is in an immunoreceptor tyrosine-based inhibitory motif, and the other is in an immunoreceptor tyrosine-based switch motif. ${ }^{72,82,83}$ To be more specific, Y248 (a PD-1 tyrosine-based switch motif) interacts with SHP-2, which is a requirement of the inhibition of proximal signaling molecules (including ZAP70, PI3K/AKT, C3G, and ERK) that consequently leads to the suppression of immune cell activation. ${ }^{79}$ In contrast, the activity of NK cells can be enhanced by histidine-rich glycoprotein, which modulates PD-1 expression via anti-C-type lectin-like receptor $1 \mathrm{~B}^{82,84}$ These findings provide potential mechanisms involved in the upregulation of PD- 1 in the peripheral blood NK cells of patients with Kaposi sarcoma, NK cells from ovarian cancer ascites, and in the peripheral and tumorinfiltrating NK cells of patients with digestive cancer. ${ }^{76,85-89}$

\section{TIGIT and CD96}

Two additional inhibitory receptors, CD96 and T cell immunoreceptor with Ig and ITIM domains (TIGIT), bind to the DNAM-1 ligand and serve to oppose DNAM- 1 function. ${ }^{90}$ TIGIT, also known as WUCAM and Vstm3, is an immune checkpoint molecule that inhibits the activation of T cells and NK cells. ${ }^{7,91-95}$ It contains an IgV domain, a transmembrane domain, and an immunoreceptor tyrosine-based inhibitory motif (ITIM). ${ }^{92}$ TIGIT has the capacity of disrupting DNAM-1 significantly through cis interactions to form heterodimers. Subsequent blockade of TIGIT with monoclonal antibodies augment the antitumor and antiviral activity of NK cells and T cells based on studies on mouse models. ${ }^{96,97}$ The expression of TIGIT plays a vital role in suppressing activation and maturation of NK cells. ${ }^{92,98-100}$ Therefore, TIGIT has a role in tumor immunosurveillance, similar to the role of the PD-1/PD-L1 axis during tumor immunosuppression. ${ }^{44}$

Studies have shown that the interaction of TIGIT with the poliovirus receptor (PVR) and poliovirus receptor-like 2 (PVRL2), also named CD112, Nectin-2, and PRR2, directly inhibits NK cell cytotoxicity. ${ }^{92,101,102}$ In addition, TIGIT has immunosuppressive effects, in that it competes with DNAM-1 for nectin-like ligands. An excellent example of the nectin-like ligand is CD155, the primary ligand for TIGIT. CD155 is expressed in many types of cancer cells. ${ }^{103}$ As highlighted, the intracellular domain of TIGIT consists of an immunoreceptor tyrosine tail (ITT) and ITIM. ${ }^{10}$ ITT-like motifs play a crucial role in inhibiting signals. The engagement of TIGIT with CD155 encourages its phosphorylation through the Srcfamily kinases Fyn and Lck; this results in the recruitment of SHIP1 , which in turn downregulates the PI3K, MAPK and NF-KB signaling pathways in modulating immune cell function. ${ }^{92,104,105}$ TIGIT can be readily detected on resting human NK cells but not on mouse NK cells. The engagement of TIGIT with CD155 prevents human NK cytotoxicity and cytokine production; this is made possible by counterbalancing DNAM-1 mediated activation, which can be reversed by antibody-mediated TIGIT blockade. ${ }^{106,107}$ The blockade of TIGIT makes NK cells resistant to inhibition by myeloid-derived suppressor cells. ${ }^{96,108}$ In like manner, a recent study showed that downregulated TIGIT expression inhibited the proliferation of colorectal cancer cells. ${ }^{37,109,110}$

CD96, also known as TACTILE (T cell activation, increased late expression), is a member of the immunoglobulin gene superfamily and an immune inhibitory receptor expressed on resting NK cells. ${ }^{111-115}$ The protein, CD96, facilitates adhesion of NK cells and T cells during immune responses. ${ }^{114}$ CD96 is similar to TIGIT, based on its competition with DNAM-1 for nectin and nectin-like ligands, and inhibits the activity of NK cells. ${ }^{116,117}$ The binding of CD96 to CD155 inhibits IFN- $\gamma$ production by NK cells. ${ }^{111,118}$ Furthermore, studies of metastatic lung tumors in the mouse model demonstrated that the antibody-mediated blockade of CD96 promoted IFN- $\gamma$ production by NK cells and improved the control of this cancer. ${ }^{11,119,120}$ The effect of antibody-mediated blockade of CD96 on NK cell function and its impact on human cancer patients remains unknown; thus, further study is needed to understand its potential as a targeted molecule for immunotherapy.

\section{TIM-3}

TIM-3 is in the TIM family of receptor proteins, all of which have similar structures. The extracellular regions of TIMs consist of a single membrane-bound distal IgV domain and a glycosylated mucin domain of variable length that is close to the membrane. ${ }^{12,121-123}$ The intracellular domain consists of a C-terminal cytoplasmic tail with five conserved tyrosine residues that interact with multiple components of the T cell receptor complex. ${ }^{124,125}$ TIM-3 is expressed on nearly all mature CD56 ${ }^{\mathrm{dim}} \mathrm{CD} 16^{+}$NK cells 
and is upregulated following stimulation with IL-12 and IL-18, and by IL-15 mediated NK cell maturation; ${ }^{126-128}$ this suggests that TIM-3 acts as a marker for NK cell maturation or activation, and TIM-3 cross-linking can inhibit NK cell-mediated cytotoxicity. ${ }^{129,130}$ Importantly, upregulation of TIM-3 has been found in gastric cancer, lung cancer, renal cancer, head and neck cancer, melanoma, schwannoma, follicular B-cell non-Hodgkin lymphoma, cervical cancer, prostate cancer, colorectal cancer, urothelial bladder carcinoma, esophageal cancer, and in the peripheral blood of patients with ovarian cancer. ${ }^{35,93,131-143}$ The coexpression of PD-1 and TIM-3 correlates with acute myelogenous leukemia progression, suggesting that TIM-3 has potential as a prognostic biomarker for various cancers.

Some of the cognate ligands for TIM-3 include galectin-9, phosphatidylserine, high mobility group protein 1, and carcinoembryonic antigen-related cell adhesion molecule $1 .^{126,144-148}$ TIM-3's cytoplasmic tail lacks a classical signaling motif and is replaced by five conserved tyrosine residues where some of them serve for signaling, and they include Y256, Y263, and Y265. ${ }^{148,149}$ The tyrosine residues signal through regulated interaction with HLA-B associated transcript-3 (Bat3). Bat3 binds to TIM-3 while in a steady-state, and sequentially recruits Lck, which is catalytically activated and promotes T cell signaling. ${ }^{150,151}$ Y256 and Y263 become phosphorylated, leading to the dissociation of Bat3; this, in turn, promotes $\mathrm{T}$ cell inhibition. ${ }^{150,151}$ TIM-3 is conservatively increased on both resting and activated T cells. In contrast, TIM-3 is only marginally increased on NK cells to oppose NK cell activation. ${ }^{152}$ Therefore, further research should better determine the role of TIM-3 in tumor surveillance to better understand its potential application in NK cell-mediated cancer treatment.

TIM-3 targeting effects do not affect non-tumor bearing tissues, although it does not seem to have any specificity. ${ }^{143}$ This remains one of the cancer research mysteries that although specificity remains a crucial factor in regulating immune responses in tissue. ${ }^{122,153,154}$ The upregulation of TIM-3 in several types of cancer raises concerns about the safety of all other tissues. However, research has shown that the safety of NK cells may be improved by irradiation. ${ }^{126,155}$ TIM-3 ligands expressed in tumor tissues, such as the Ceacam-1 and galectin-9. The effect of the TIM-3 blockade on tumor tissue is preferential. ${ }^{131,156}$ Therefore, targeting TIM-3 in a person with neck cancer will only affect other tissues if those tissues have some known ligands of the TIM-3 blockade. ${ }^{157}$ TIM-3 blockade is regulated by functional specification, a concept through which pathways such as the TIM-3 pathway sometimes regulate immune responses' distinct features; thus, some other tissues in a patient with the neck cancer can remain unaffected by the targeting. ${ }^{13,156,157}$

\section{CD112R}

CD112R, also known as poliovirus receptor-related immunoglobulin domain protein (PVRIG), is a newly identified inhibitory checkpoint receptor in the PVR-like family. CD112R is constitutively expressed on mouse NK cells, natural killer $\mathrm{T}$ cells, and $\mathrm{CD} 8^{+} \mathrm{T}$ cells, and also expressed on human T cells and NK cells. ${ }^{101,158-160}$ Based on research findings, the expression of CD112R on CD16 ${ }^{+}$NK cells from human PBMCs was nearly equal to that on CD16 ${ }^{-}$NK cells. ${ }^{160}$ CD112R was also highly expressed on NK cells from ovarian, endometrial, kidney, prostate, lung, and breast cancers. ${ }^{11}$ The receptor interacts with PVRL2 but not PVR (CD155) and competes with CD226 for PVRL2 binding to inhibit $T$ cell and NK cell function. ${ }^{11,158,160}$ Human tumors with high PVRL2 expression and lower PVR expression are more likely to respond to CD112R blockade ex vivo. ${ }^{11}$

In the CD112R-deficient mouse TME of colon carcinoma and melanoma, increased $\mathrm{CD}^{+} \mathrm{T}$ cell effector function inhibited tumor growth compared with wild-type mice. Furthermore, it has been established that anti-CD112R antagonists combined with anti-PD-L1 could also reduce tumor growth or metastasis. ${ }^{155,158,161,162}$ In an ex vivo study on human tumor-infiltrating lymphocytes, CD112R blockade enhanced T-cell function, and in combination with TIGIT or PD-1 blockade, further enhanced the effect. ${ }^{11,163}$ However, its function in human NK cells is still inadequately known. In a preclinical study, it was established that the blockade of CD112R alone or together with TIGIT-blockade enhances the trastuzumab-triggered antitumor response in human NK cells and improves the efficacy of trastuzumab therapy for breast cancer. ${ }^{104,160,164}$ This finding suggests that CD112R on NK cells may be a potential target for breast cancer treatment in the future, although its efficacy and safety remain to be further verified through animal experiments and clinical trials.

\section{IL-1R8}

Interleukin-1 receptor 8 (IL-1R8) is also called a single immunoglobin IL-1-related receptor and is a member of the IL-1 receptor family. It has functional and structural characteristics that negatively regulate ILR and Toll-like receptor. ${ }^{75,165,166}$ IL-1R8 has been identified as a checkpoint protein in NK cells that regulate antitumor activity in solid cancers. The peripheral blood of $\| 1 \mathrm{r}^{-/-}$ mice proved to have an increase in NK cell number and maturity. The attenuation of IL-18 destroyed NK cell maturation in $111 \mathrm{r}^{-/-}$ cells. $^{75,167}$ The deficiency of IL-1R8 can also result in enhanced IL18-dependent activation of the JNK and mTOR pathways essential for the control of NK cell differentiation, activation, and metabolism. NK cells with low IL-1R8 levels tend to sustain more extended activation after stimulation. These NK cells also indicate high antitumor immunity levels (like IFN- $\gamma$ ); whereas, small proinflammatory cytokine (CXCL1, IL-6, CCL2, IL-1 $\beta$, and TNF- $\alpha$ ) levels attribute to promoting tumor growth. ${ }^{142,167,168}$ Importantly, a genetic IL-1R8 blockade can result in NK cell-mediated resistance to hematogenous liver metastasis, hepatic carcinogenesis, and lung metastasis. ${ }^{108,167}$ Interventions to overcome this resistance are already in their various testing stages; chimeric antigen receptors (CARs) engineered NK cells are one of them that has been found to be sufficiently effective. ${ }^{169-173}$ These findings show that IL-1R8 is an NK cell checkpoint protein that may be inhibited to promote antitumor activity.

\section{LAG-3}

Structurally, LAG-3 is similar to CD4, yet it manifests a higher binding affinity to MHC class II molecules than CD4. It is expressed on activated T cells and NK cells. ${ }^{174}$ Another of its potential ligand is LSECtin, a member of the DCSIGN family. ${ }^{175,176}$ There is a high expression of LAG-3 in patients with Hodgkin lymphoma, acute myelocytic leukemia, and chronic lymphocytic leukemia. ${ }^{177-179}$ Its cytoplasmic tail is made up of three unique and conserved regions in mice and humans. It includes a serene phosphorylation site, a glutamic-acid proline (EP) repeats, and a KIEELE motif. Of the three, the KIEELE motif is the one that serves as the inhibitory function of LAG-3 in CD4 ${ }^{+}$cells. ${ }^{180}$ The effector function of T cells is inhibited by the engagement of LAG-3 and enhanced by the blockade of LAG-3. ${ }^{180,181}$ It is interesting to learn that LAG-3 is involved in the exhaustion of T cells as a result of pathophysiological basics; hence its combination with PD-1 synergizes to reinstate $T$ cell function. ${ }^{182,183}$ The role of LAG-3, however, in regulating NK cell function is not yet clear and therefore calls for more research and investigation. NK cells from LAG-3 deficient mice show defects in the killing of specific cancer cells. ${ }^{184}$ It is worth noting that blocking the LAG-3 pathway with the use of LAG-3 antibodies or even soluble LAG-3 does not have any effect on the cytotoxicity of human NK cells. ${ }^{176,185}$ Even so, targeting LAG-3 may be useful in immunotherapy due to its influence on $T$ cell and NK cell effector function.

\section{NKG2A}

NK group 2 member A (NKG2A) is a NK cell receptor of the NKG2 family, a type II membrane receptor that forms a heterodimer with CD94. ${ }^{16,28}$ It dimerizes with CD94 to form an inhibitory receptor 
that is related to C-type lectins and recognizes HLA-E (also known as $\mathrm{MHC}$ class I antigen E). ${ }^{28}$ These inhibitory receptors interact with MHC I ligands on target cells, leading to complete inhibition of NK cell granule polarization and the prevention of cytotoxic granule release. ${ }^{186}$ NKG2A contains two immunoreceptor tyrosinebased inhibition motifs (ITIMs) in its cytoplasmic tail. These ITIMs are phosphorylated following ligation of the ITIM-bearing receptor and facilitate this leads to the recruitment of tyrosine phosphatases, such as $\mathrm{SH} 2$ domain-containing phosphatase (SHP)- 1 and SHP-2. ${ }^{187}$ The recruitment of SHP-1 by the ITIM-bearing receptors seems to inhibit the initiation of signaling in that it blocks most downstream signals in NK cells. ${ }^{187}$ The selective dephosphorylation of Vav1, the only protein detectably associated with the catalytic site of SHP-1, by SHP-1 leads to the inhibition of NK cell cytotoxicity, whereas the phosphorylation of an adapter protein CRK leads to the inhibition of NK cell function after ligation of NKG2A to HLA-E. ${ }^{188-190}$ Previous studies have shown that NK cells express NKG2A in cancers of the breast, cervix, liver, and lung. ${ }^{191-194}$ Importantly, tumor immunity increases when the inhibitory NKG2A receptor is blocked. The strong capability of NKG2A to suppress NK cells means that the blockade of NKG2A will effectively lead to the restoration of NK cells. For example, the blockade of NKG2A in an experiment using an anti-NKG2A antibody against NKG2A ligand in mice led to the effective alleviation of the functional impairment of NK cells. ${ }^{98,195}$ The prospective use of immune checkpoint inhibitors, as demonstrated by NKG2A, propels the development of cancer immunotherapy. ${ }^{42,196-198}$

\section{ACTIVATING IMMUNE CHECKPOINT RECEPTORS}

Some additional co-stimulatory pathways are involved in regulating NK cell responses, which impede the development of cancer and contribute to cancer immunotherapy. For example, CD226, also known as PTA-1 or DNAM-1, is an activating immune receptor that is expressed on NK cells, which has cytolytic activity and functions in lymphokine secretion. ${ }^{116}$ It contains two Ig-like domains on its extracellular portion and has a cytoplasmic tail containing three tyrosine residues. CD226 is in the immunoglobulin superfamily and modulates NK cell functions by interacting with PVR, Nectin-2, CD96, and TIGIT. ${ }^{84,199-203}$ In murine models, downregulation of CD96 enhanced antitumor functions of CD226, thereby reducing lung metastases and tumor growth. These findings indicate that CD226 has potential as an activating checkpoint receptor for immunotherapy.

NKG2D is an activating immune receptor expressed on NK cells that can trigger cytotoxicity and is in the CD94/NKG2 family of Ctype lectin-like receptors. ${ }^{200,204}$ NKG2D binds to its ligands that occur on the surface of tumor cells, such as stem-like tumor cells, and alters these cells to be more susceptible to immune destruction. $^{205,206}$ Alternatively, tumor cells can evade immune surveillance by shedding soluble NKG2D ligands. Recent studies showed that NKG2D targeting might be a practical immunotherapeutic approach for the treatment of cancer. ${ }^{203,207,208}$ Inducing activating receptors and their ligands can be targeted by some potential strategies such as NKG2D ligand a3 domain-specific antibodies, cytokines (IL-2, IL-15, and IL-21), histamine, anti-TGF- $\beta$ monoclonal antibodies, TGF- $\beta$ receptor I kinase inhibitors, TGF- $\beta$ antisense oligonucleotides demethylating agents (5-azacytidine and 5-aza-2'-deoxycytidine) (Fig. 3). ${ }^{209-216}$ In particular, targeting the NKG2D/soluble MIC, which is highly expressed in many malignant carcinoma cells (like melanoma and leukemia), maybe a potential approach for the treatment of these cancers.

NK CELL AS A PROMISING THERAPEUTIC TARGET FOR CANCER NK cells belong to the innate immune system and are active in the early fight against cancer through their ability to kill abnormal cells. ${ }^{101,217}$ They do not require previous stimulation and can induce an effective antitumor response that is mainly mediated by specialized cell-surface receptors, indicating malignancy. ${ }^{218}$ There is evidence from preclinical experiments and clinical trials for the development of NK cell-targeted immunotherapies (Tables 1 and 2) as a solution to chemo-resistant cancers, such as non-Hodgkin lymphoma. ${ }^{80,219}$ Monoclonal antibodies (mAbs) have been shown to elicit or augment prevailing antitumor immune responses. Their activity is referred to as 'checkpoint blockade mAbs' and is based on the principle of disturbing suppressive signals made by inhibitory receptors of lymphocytes. Recent methodologies have also been developed using the mAb-mediated blockade of definite NK cell immune checkpoints. ${ }^{48,52,74,157,220}$

The restoration of NK cell activity against $\mathrm{HLA}-\mathrm{I}^{+}$tumor cells is essential in the therapeutic targeting for novel immunotherapies that depend on therapeutic monoclonal antibodies, such as antipan-KIR2D. ${ }^{67,221,222}$ In the absence of infection, inhibitory HLA-KIR signals dominate and protect tumor cells from NK cell-mediated lysis, while intracytoplasmic binding of KIR2DL or KIR3DL with ITIMs prevent NK cell lysis activity. ${ }^{68,223}$ Conversely, KIR2DS and KIR3DS are noncovalently associated with an ITAM-bearing adapter molecule DAP-12, which results in phosphorylation of tyrosine residue in the ITAM and recruiting ZAP-70 or Syk, leading to cellular activation and increases the capacity of NK cells to recognize tumor cells; this is an essential target for cancer treatment. ${ }^{26,224,225}$ KIR has been established to play a critical role in mediating self-tolerance along with the facilitation of cytotoxicity against infected cells and transformed cells. According to research, KIR-HLA relationships can be linked to the incidence and course of both solid tumors and hematologic malignancies. ${ }^{67,226}$ The inhibitory KIR-HLA relationships have been established as being overrepresented in patients with acute and chronic leukemia, breast cancer, Hodgkin lymphoma, and melanoma. ${ }^{226}$ The assertion can be understood based on matched autologous inhibitory KIR-HLA interactions preventing the lysis of cancerous target cells. ${ }^{227}$ Autologous NK cell therapy, which is a novel expansion method and will help patients at the advanced stage of digestive cancer, is in phase I trial. ${ }^{227}$ As part of targeting cancer therapies, the expression of activating KIRs in different circumstances can be linked to better outcomes in some malignancies. ${ }^{26}$ KIR-ligand modulation is vital for cancer therapy as exemplified by the initial therapeutic manipulation of KIR-ligand relationships in the case of $T$ cell-depleted, haploidentical hematopoietic stem cell transplantation for hematologic malignancies. The inhibitory KIR-ligand relationship has been established as important in the realization of NK cell alloreactive potential with special attention on the balance of activating and inhibitory signals that direct cytotoxicity. ${ }^{26,228}$

Preclinical studies and clinical trials of PD-1 blockade therapy have led to impressive results in mediating tumor eradication. As discussed, NK cells secrete pro-inflammatory cytokines that promote the expression of PD-L1 in tumor cells and enhance the inhibiting role of PD-1. At the same time, PD-1 antibodies can bind to NK cell surface PD-1, prevent NK cell depletion, and enhance NK cell antitumor response. ${ }^{229,230}$ Pidilizumab (previously CT-011) is an anti-PD-1 antibody that recently entered clinical trials. Pidilizumab can enhance human NK cell activity against autologous primary multiple myeloma cells. ${ }^{4,231-233}$ In addition, highly dense activated human primary NK cells can kill colorectal carcinoma cells grown in 3D cultures independent of PD-L1 expression, suggesting that the use of allogeneic activated NK cells could be an effective treatment of this cancer. ${ }^{38,77,93,234-236}$ Immune evasion via PD-1/PD-L1 in NK cells and monocytes/ macrophages is prominent in Hodgkin's lymphoma. $38,77,93,234-237$ Also, one of the immune checkpoint molecules that have already been employed in specific preclinical tumor models is the establishment of efficacy in blocking TIGIT to modulate NK cell function and has resulted in improved clinical responses in patients with cancer. ${ }^{7,94,95}$ 


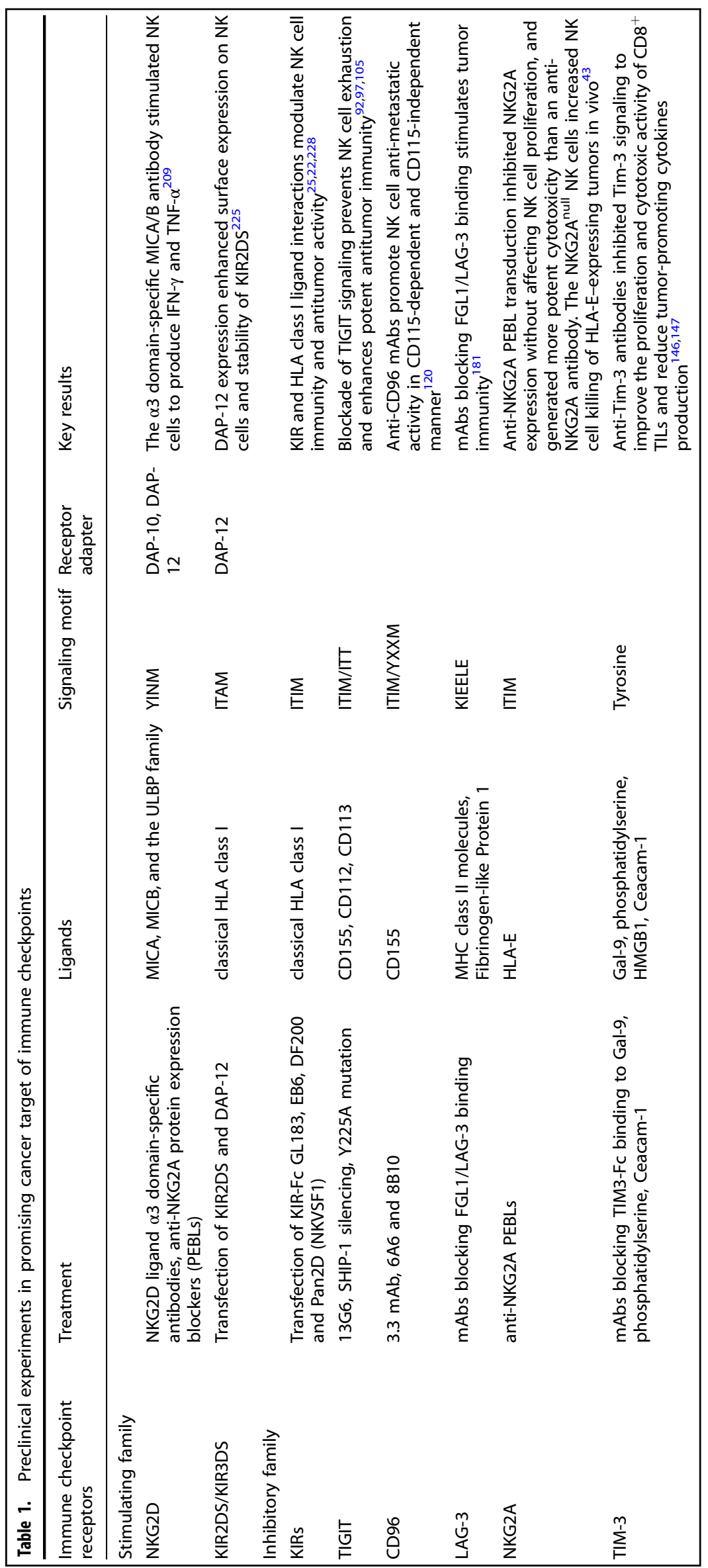




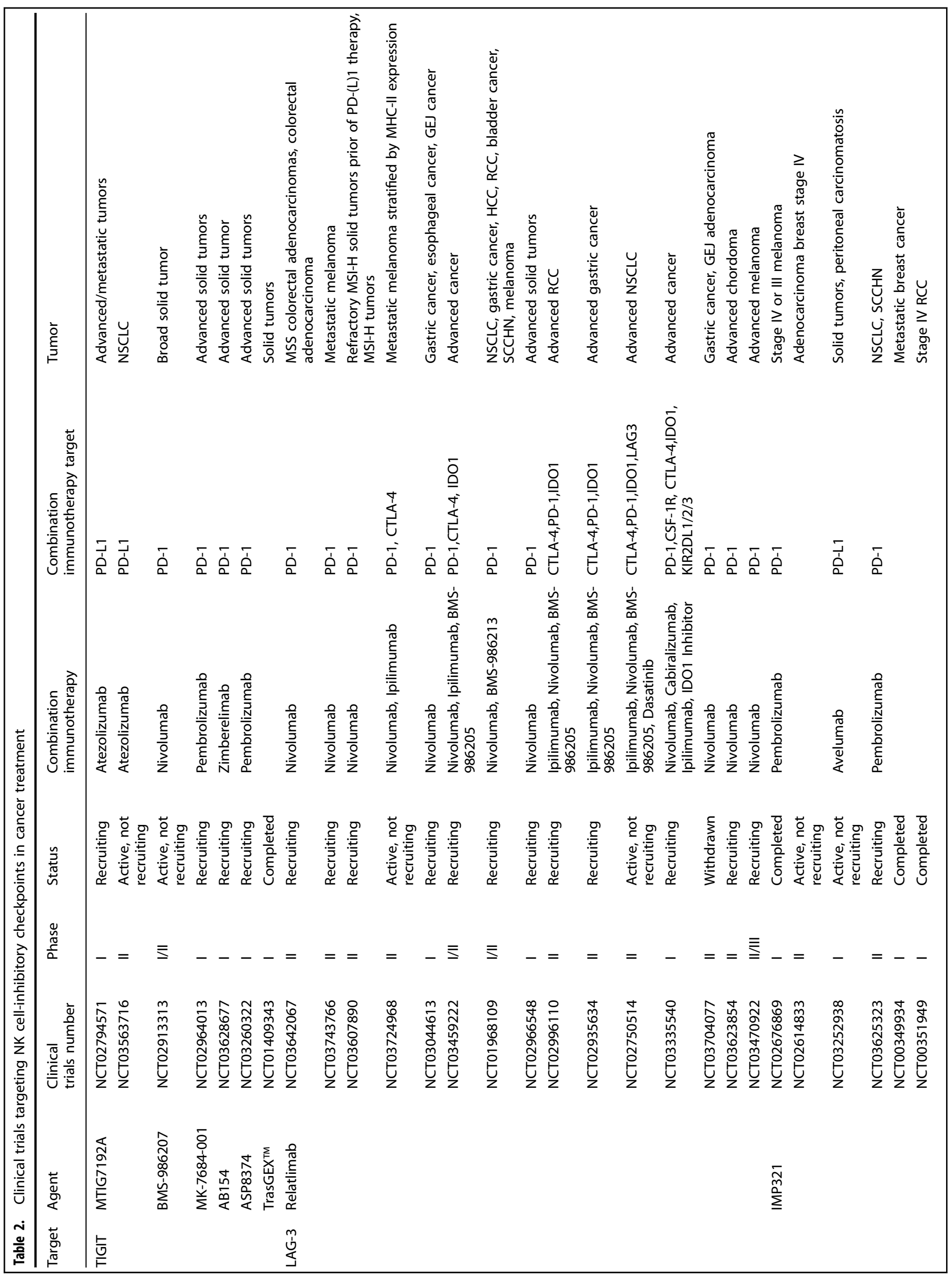




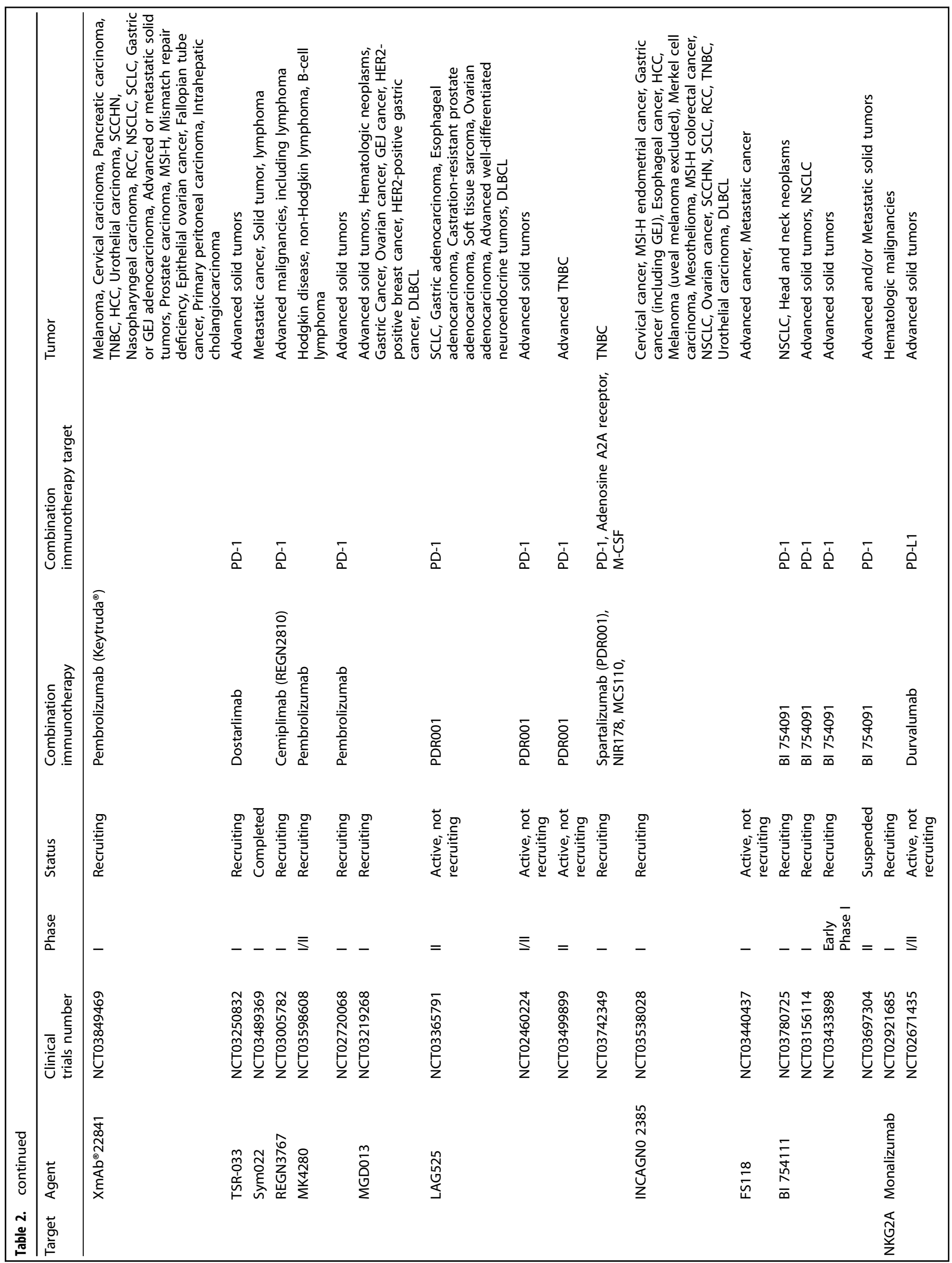




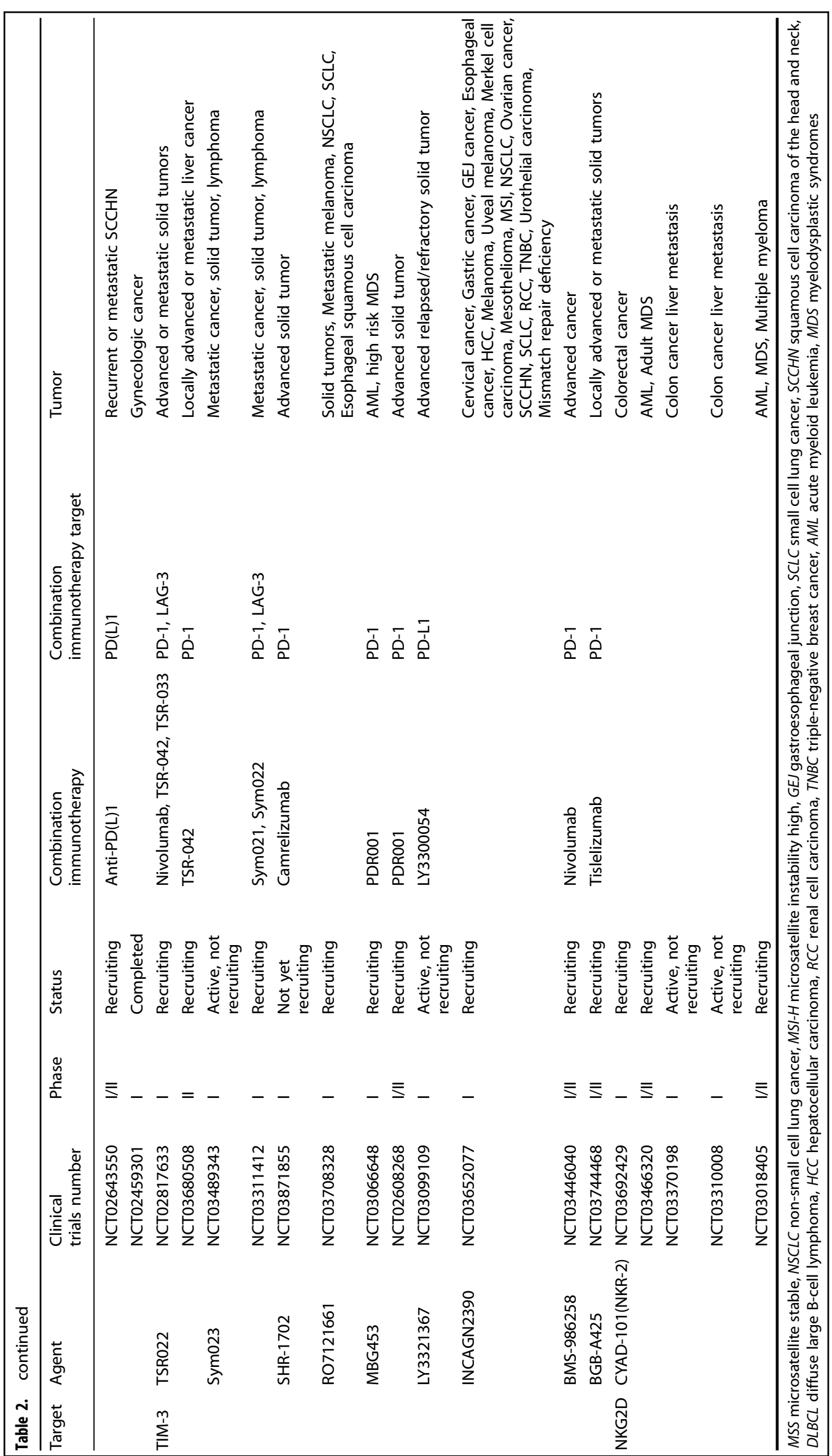


There are ongoing phase I/II trials of the application of NK cells combined with PD-1 antibodies (sintilimab and pembrolizumab) in the treatment of patients with advanced non-small-cell lung cancer (NCT03958097) and advanced biliary tract cancer (NCT03937895). Thus, there are many ongoing studies of immunotherapies for PD-1 blockade by targeting NK cells in cancer treatment.

Lirilumab targeting pan-KIR2D and monalizumab targeting NKG2A have been demonstrated to disrupt the interactions of inhibitory KIR on NK cells with classical HLA class I-peptide complex on tumor cells or NKG2A with nonclassical HLA-I molecular HLA-E, which could unleash the antitumor NK cell cytotoxic activity mimicking "missing-self" response. ${ }^{16,69,238}$ Both lirilumab and monalizumab are safe with limited side effects upon prolonged treatment in phase I clinical trials. ${ }^{237}$ Markedly, all dosages of lirilumab induced high levels of free KIR2Ds (>95\%). ${ }^{237}$ These agents are currently undergoing phase I/II clinical trials across a range of hematologic and solid tumors in monotherapy or combination with other immune checkpoint blockades, including PD-1 inhibition (NCT02671435 and NCT02643550). ${ }^{239}$

The role of NK cells in cancer therapy also promotes the activating receptor CD226, which is a mediator of the cells' responses against tumors. Although, the mechanism by which the CD226 receptors exert control over the function of NK cell is a challenge, the engagement of the receptor with CD155 in the transcription factor FOXO1 phosphorylation, which results in the inactivation of the receptor's negative regulatory control over the effector function of NK cell. ${ }^{226,240}$

As discussed, the activated NKG2D receptor is a critical NKG2 family receptor on NK cells and can bind to NKG2D ligands expressed on tumor cells. This binding allows NK cells to activate and destroy tumor cells, as well as produce cytokines. The NKG2D/ NKG2D ligand axis has been recognized to play an essential role in antitumor activity. Infusions of activated NK cells can scavenge circulating soluble MICA in cancer patients, leading to reinstate NKG2D-mediated immune surveillance. ${ }^{235}$ Although our understanding of the control of NKG2D ligand expression remains limited, research in recent years revealed various cellular mechanisms by which cancer cells evaded detection by reducing the "stress-induced ligands" expression. Recently, NKG2D ligand (MICA and MICB) a3 domain-specific antibodies were developed to restore NK cell-mediated tumor immunity by increasing the density of stimulatory MICA and MICB ligands on the surface of tumor cells along with reducing the shedding of both MICA and MICB. $^{236}$ Therefore, targeting co-stimulatory receptor signaling may serve as an alternative strategy to boost NK cell function and prove beneficial for cancer treatment (Fig. 3).

The difference between the current treatment strategies and the promising immune checkpoint molecules such as TIGIT in NK cells is that they can be modulated to enhance clinical responses. The diverse receptors expressed on the tumor-infiltrating surfaces of NK cells would increase the binding of tumors once activated and destroy them. ${ }^{121}$ The establishment of the receptors, especially the IL-1R2 and IL-1R8, which are effective immune regulators, will improve immune surveillance and control. ${ }^{165}$ Single immunoglobulin IL-1R-related receptor, SIGIRR, also known as TIR8 or IL-1R8, PD-1, LAG-3, CTLA4, and TIM-3 has distinct functional and structural characteristics that adapt it better to regulate the expression of NK cells in a TME. ${ }^{13,241}$ Other receptors that can complement the role of NK cell subsets to increase their prediction of malignancy are TIM-3 and IL-15. ${ }^{154,242}$

Given different cancer types and tumor staging, it is hard to say which is better for restoring NK cell-mediated tumor immunity between targeting the NKG2D receptor and targeting KIR or NKG2A. Although one recent in vitro study found blocking the inhibitory signal (blocking of HLA-KIR) is not so efficient as the activating signal increases (Ara-C treatment that induced NKG2D ligands) potentiate NK cell responses against acute myelocytic leukemia (AML) cells, more in vitro and in vivo studies are needed to demonstrate it. However, this study provides evidence that a combination of both treatments has better efficiency. ${ }^{243}$

Recently, adoptive cell therapy strategies, including cancertargeting CARs-engineered NK cell infusion have been explored in preclinical and clinical studies. ${ }^{169-173}$ Chang et al. demonstrated a chimeric receptor with the NK cell-activating molecule NKG2D, plus two vital signaling molecules, DAP-10 and CD3Z, enhanced the antitumor effect of peripheral blood NK cells on osteosarcoma. ${ }^{169}$ More recently, human induced pluripotent stem cells (iPSCs) have been proven to be more efficient in genetically modifying and expressing CAR constructs specifically designed to enhance NK cell antitumor activity. ${ }^{244}$ To date, CAR engineered NK cells, which are from autogenic or allogeneic peripheral blood mononuclear cell, umbilical cord blood, human embryonic stem cell, human iPSC, and NK cell lines, ${ }^{244-254}$ are at the beginning but greatly promising in tumor immunotherapy.

The increasing resistance of several cancers to chemotherapy demands a clinical strategy to increase immune checkpoint molecules' effectiveness. Findings from a study indicated that the elimination of regulatory $T$ cells (Treg) using IL-2-diphtheria fusion would have a therapeutic benefit if haploidentical NK cells are infused in AML patients. The study shows that Treg cells have a negative role in population expansion of NK cells following transfer. In this first clinical trial, an administration of recombinant IL-15 clearing lung cancer lesions in metastatic cancer patients astatic further supports the effectiveness of immune checkpoint molecules in NK cells as target cancer immunotherapy. ${ }^{255}$ Combined therapy is emerging more effective strategy for treating cancer. Although targeting NK cells is a leading intervention cancer immunotherapy through their direct lysis of tumor cells, the intervention's effectiveness is reduced by immune evasion. ${ }^{256-258}$ Combination therapy provides an alternative strategy for increasing the effectiveness of NK cells' antitumor ability. ${ }^{55}$ One of the target combinations is NK cells with nanomaterials and oncolytic viruses. TIGIT combined with CD112 and CD155; blocking CD96 is considered another promising therapeutic role. ${ }^{259}$ Research findings have proved that a combination of TIGIT blockade and anti-PD-1/PD-L1 antibodies forms a synergy that may be used in preclinical trials. ${ }^{156}$ Other findings from a recent study indicated that the checkpoint receptor TIGIT blockage prevents the exhaustion of NK cell and triggers the elicitation of T-cell immunity that are potent tumorspecific in a NK cell-dependent manner. ${ }^{19,260}$ A combination of engineering NK cells with specific immune checkpoint signaling domains also provides a promising therapeutic potential for the treatment of recurrent and refractory cancer.

\section{STUDY AND PROGRESS OF ADENOSINE IN THE NK CELL CANCER IMMUNOTHERAPY}

To complement the general role of control and surveillance of malignant cells that the immune system play, the development of adoptive cellular therapy and checkpoint blockade has brought about a revolution in the landscape of cancer therapy and promises the potential in which oncologists can utilize a patient's immune system to treat cancer. ${ }^{185,261-263}$ Purinergic signaling axis has gained prominence as one of the mechanisms of tumormediated immunosuppressions, in which the purine nucleoside adenosine produced in the TME has the ability of potently suppressing NK cell function. ${ }^{264-266}$ During this event, the production of extracellular adenosine is triggered by the mediation of the cell surface ectoenzymes CD38, CD39, and CD73, ${ }^{267-269}$ and the development of therapeutic agents has focused on targeting these enzymes as responses. ${ }^{270}$ The therapeutic agents also target receptors, including the downstream adenosine receptors (AR) such as $A_{1} R, A_{2 B} R, A_{2 A} R$, and $A_{3} R$, to promote antitumor immune responses. ${ }^{16,264,271}$ 


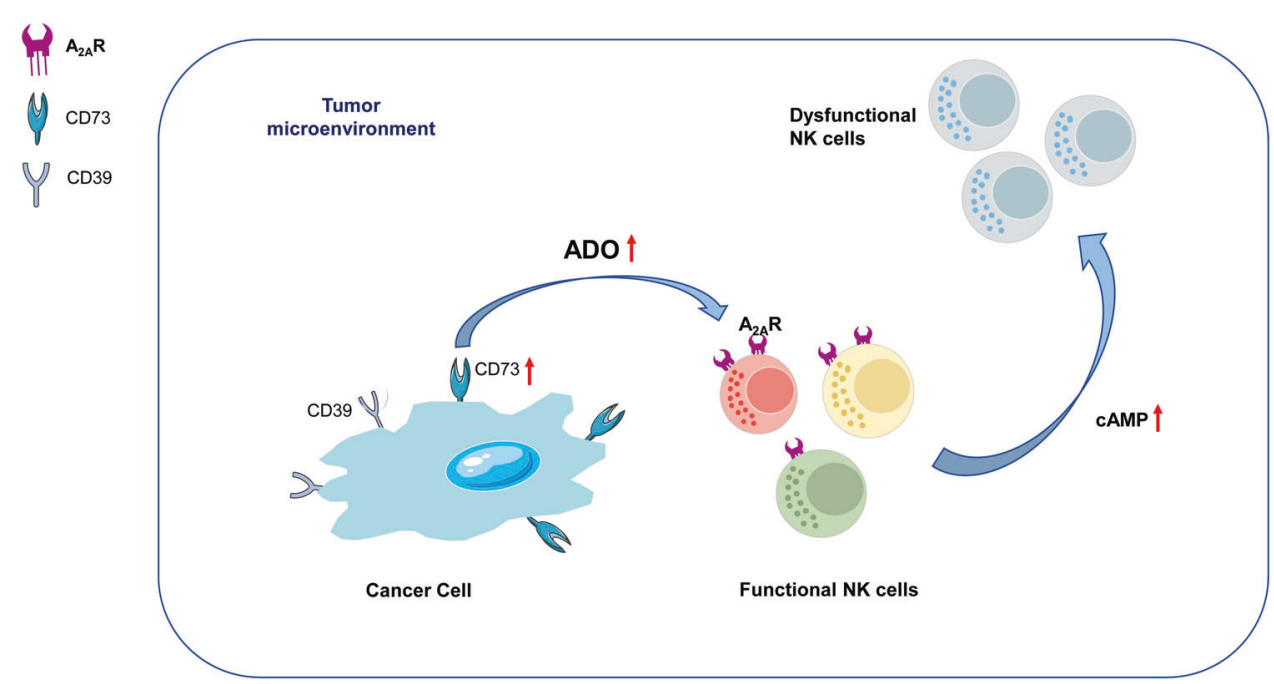

Fig. 4 Adenosine-axis in the tumor microenvironment mediates immune suppression of NK cells. In the tumor microenvironment, stress such as hypoxia increases extracellular ATP levels and CD73 expression on cancer cells, leading to adenosine (ADO) upregulation and the activation of downstream signaling through $A_{2 A} R$ on NK cells. $A D O / A_{2 A} R$ signaling results in dysfunction of NK cell metabolic and effector functions

In NK cells, the signaling of adenosine (ADO) signals via $A_{2 A} R$ suppresses their cytokine production and cytotoxicity function. ${ }^{272,273}$ ADO signaling plays a critical role in immune regulation, as further underscored by the NK cells total dysfunction in individuals presenting with a variant of severe combined immunodeficiency caused when adenosine deaminase (ADA) undergoes a mutation that catalyzes ADO conversion to inosine. ${ }^{262,274,275}$ Also, the expression of CD38 on tumors, T cells, and NK cells promote the generation of ADO followed by proliferation and suppression of $\mathrm{T}$ cell function. In addition to suppressing T cell responses, CAMP has an overall inhibitory effect on NK cells. ${ }^{264,276-278}$

NK cells express high $A_{2 A} R$ levels, specific $A_{2 A} R$ agonists, or ADO suppressed NK cells due to their cytokine production and cytotoxic function. ${ }^{142,279-281}$ Intracellular cAMP concentration enhancement mediated by $A_{2 A} R$ is believed to be the predominant mechanism explaining how ADO suppresses NK cell activity (Fig. 4). ${ }^{264,282,283}$ Like T cells, NK cells express the $A_{3} R$, the function of which has been found to regulate $A_{3} R$ positively and NK cells, resulting in metastatic and primary tumor growth both in human and mouse models of melanoma and colon cancer. ${ }^{284}$

There are several trials on approaches involving a combination of CD38 with anti-PD-1 that have been found to stimulate enhanced antitumor responses by $T$ cell-mediated by enhanced Granzyme B and IFN- $\gamma$ expression by $\mathrm{CD}^{+} \mathrm{T}^{+}$cells. $^{79,264,285}$ Findings from another study showed that $A_{2 A} R$ cells had enhanced the penetration in hypoxic tumors. ${ }^{286}$ Although the focus of most of the combination approaches indicates the possibility of ADO targeting aimed at enhancing T cell responses, ADO axis targeting, including NK cells, can enhance other immune subsets comprised in the TME. ${ }^{287}$ The accumulation of ADO predominantly in the TME when the extracellular ATP is catabolized to ADO by CD38, CD39, and CD73 is expressed in immune and tumor cells, and it has been reported that ADO mediates the suppression of antitumor immunity when the ADO receptors. $^{185,268}$

Extracellular ADO production in the TME

TME forms site for high ADO concentrations caused by inflammation, tissue disruption, and contribution of stromal and immune cells. ${ }^{288,289}$ Hypoxia is another predominant driver of increased ADO concentration through stimulation of large ATP formation. ${ }^{290}$ It also contributes to the expression of HIF-1a, a well-defined transcription factor, which stimulates the expression of ectoenzymes CD73 (5'-NT) and CD39 (NTPDase1) on stromal cells, tumor cells, as well as tumor-infiltrating immunosuppressive cell subsets, for example, myeloid-derived suppressor cells and Treg cells. ${ }^{242,291}$ Part of the evidence to support the role of CD39 and CD73 in tumorigenesis and inflammation is that mice deficient in CD73 or CD39 are susceptible to autoimmunity/inflammation and be tumor resistant as a result of ADO-mediated immunosuppression alleviation. ${ }^{292-294}$

Targeting the adenosine pathway in TME to improve immunotherapies

Cancer immunotherapies are currently considered the fourth pillar in the treatment of cancer. ${ }^{295}$ Several research studies on the role of $A D O$ negatively regulating NK cell responses through $A_{2 A} R$, and naturally targeting the ADO pathway are part of cancer immunotherapy interventions that may further boost the efficacy of approved immunotherapies in the clinic. ${ }^{35,267,296,297}$ Although ADO has been found to thwart the antitumor immune responses that radiotherapy elicits, when ADO receptors are antagonized in cancers, the approach allows the interruption of the ADOdependent immune evasion that results in antitumor immune responses. ${ }^{298}$ The immunosuppressive role of NK cells, combination strategies rationally targeting the pathway with adoptive cell therapies (ACT), and checkpoint inhibitors can synergistically promote the function of antitumor immune cell function, a process that utilizes ATP generated in the TME (Fig. 4) (Table 3). ${ }^{271,299,300}$

\section{LIMITATIONS OF IMMUNE CHECKPOINT MOLECULES IN NK CELL FOR CANCER IMMUNOTHERAPY}

Age is the main contributor to NK cell altered expression of checkpoint molecules in NK cells. ${ }^{47,301}$ Activating receptors alteration has been associated with chronic exposure of the receptors to ligands on tumor cells. ${ }^{302}$ There is also a synergistic effect between age and cancer that diminishes NK cell-mediated tumor immunosurveillance, which may also be associated with NK cell immunoresistance. ${ }^{257,303}$ However, oncologists have made significant successes in developing different strategies to harness the power of NK cells aimed at targeting tumor cells, such as adoptive transfer therapy, which involves allogeneic or autologous expanded NK cells. ${ }^{2,304}$ 


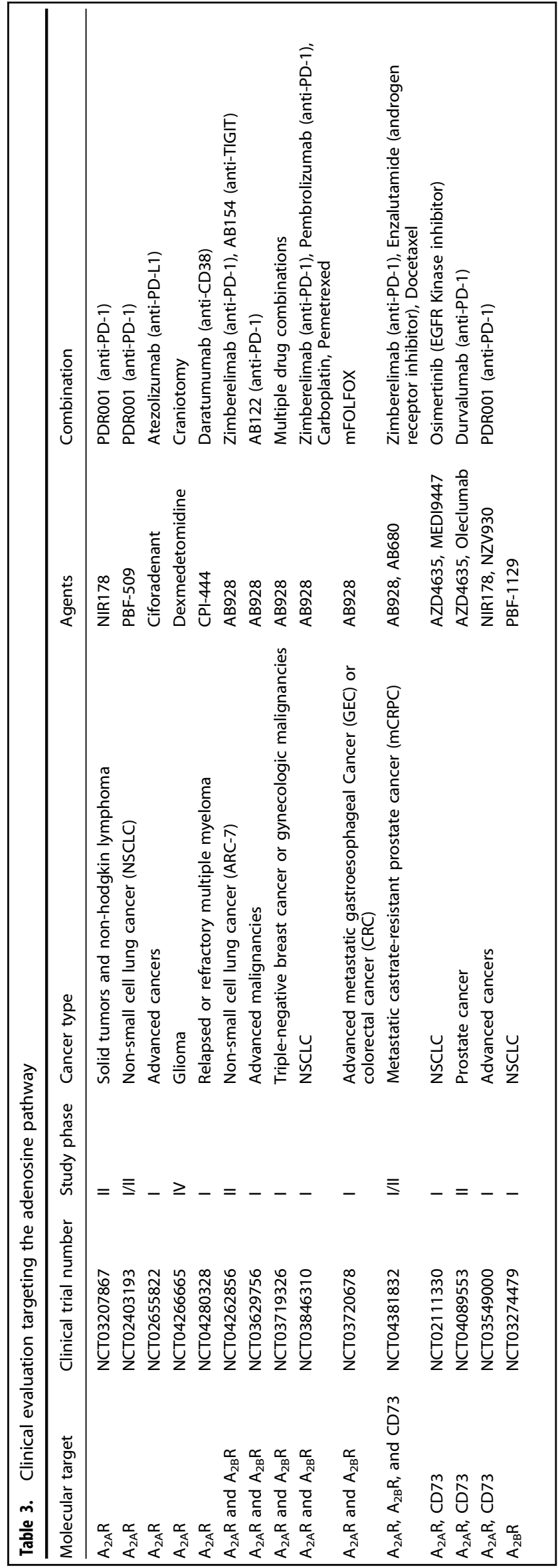

\section{FUTURE DIRECTIONS TO STUDY THE NK CELLS IN CANCER} IMMUNOTHERAPY

Although numerous trials have been carried out on various innovations on cancer therapy, cancer treatment is still a significant challenge. Proposals by academia, such as identifying allogeneic NK cells as one of the most effective cancer treatment options, have not made much progress in identifying a cancer therapy applicable to a majority of cancers without issues such as NK cell receptor downregulation. ${ }^{305}$ In my opinion, leaving these studies the academia and biotech companies would not bring a solution any time soon. I propose that the World Health Organization developed governments and other non-governmental organization fund multi-disciplinary groups and organizations in the healthcare field to conduct researches aimed at both preventive and curative recommendations for cancer therapy, especially for stage I to III treatment strategies for non-small cell lung cancer, in which radiotherapy and surgery are usually employed. ${ }^{306}$ Therefore, considering combinatorial approaches drawing together different treatment strategies that involve NK cell functions would be worthwhile. NK cell research companies should consider developing NK cell-specific treatments underlying scientific findings and principles of their best product pipelines, demonstrating highly innovative concepts and practices that herald future sustainable and cost-effective clinical applications.

Since the role of NK cell-based immunotherapy strategies has gained immense support as a proven intervention against cancer, I advise that everyone should not consider it as the bottom-line solution based on which all future research will focus. NK cells can only be effective in a few types of cancer, considering their complex networking, heterogeneity, as well as the inherent adaptability of a wide range of tumors that evade destruction by immune cells. In the meantime, future studies should focus resources on improving the efficacy of NK cell products that are currently available. ${ }^{94}$ The discoveries of all NK cell-based immunotherapies should proceed to clinical implementation. ${ }^{306}$

\section{CONCLUSION}

Human NK cells are essential and play a significant role in the development of therapeutic treatments for cancer. In the tumor microenvironment, NK cells transform to become dysfunctional cells with enhanced expression of inhibitory immune checkpoints, including the non-HLA-class I-specific inhibitory receptors (such as PD-1, TIGIT, CD112R, CD96, IL-1R8, and TIM-3) and HLA-class Ispecific inhibitory receptors (such as KIR, NKG2A, and LAG-3), as well as decreased expression of activating receptors (such as $N K G 2 D$ and $C D 226)$. Adenosine- $A_{2 A} R$ signaling is emerging as a novel inhibitory immune checkpoint pathway in NK cells. Blockade of these inhibitory checkpoint molecules with the use of immune receptor inhibitors, as well as activation of co-stimulatory receptor signaling by inducing co-stimulatory receptor expression, can reinstate the antitumor activity of NK cells. Many primary researches support the efficacy of NK cell-targeted immunotherapies based on immune checkpoint molecules in NK cells at preclinical phase and are ongoing at different clinical phases. In addition, more research is needed on the safety, tolerability, and clinical efficacy of drugs targeting these checkpoints and their combination therapy. There is a need to identify highly effective strategies of overcoming immune resistance, the main challenge in cancer immunotherapy, such as through CAR engineered NK cells. Above all, an in-depth understanding of immune checkpoint molecules that target NK cells could help develop new therapeutic strategies against refractory cancers.

\section{ACKNOWLEDGEMENTS}

This review was funded by the National Natural Science Foundation of China (No. 81400659, 81974377), Natural Science Fund of Liaoning Province (No. 2017225032, 
20180551193, 2020-MS-181), Shenyang Science and Technology Project (No. 17-2309-16), and the 345 Talent Project of Shengjing Hospital (No. 40B).

\section{AUTHOR CONTRIBUTIONS}

Conceptualization: C.D., R.S., and F.X.; writing-original draft preparation: Y.C. and X. W.; writing-review and editing: T.J., Y.T., C.W., R.S., and F.X.

\section{ADDITIONAL INFORMATION}

The online version of this article (https://doi.org/10.1038/s41392-020-00348-8) contains supplementary material, which is available to authorized users.

Competing interests: The authors declare no competing interests.

\section{REFERENCES}

1. Mandal, A. \& Viswanathan, C. Natural killer cells: In health and disease. Hematol. Oncol. Stem Cell Ther. 8, 47-55 (2015).

2. Seki, S. et al. The liver as a crucial organ in the first line of host defense: the roles of Kupffer cells, natural killer (NK) cells and NK1.1 Ag ${ }^{+} \mathrm{T}$ cells in $\mathrm{T}$ helper 1 immune responses. Immunol. Rev. 174, 35-46 (2000).

3. Muraro, E. et al. Improved Natural Killer cell activity and retained anti-tumor $\mathrm{CD}^{+} \mathrm{T}$ cell responses contribute to the induction of a pathological complete response in HER2-positive breast cancer patients undergoing neoadjuvant chemotherapy. J. Transl. Med. 13, 204 (2015).

4. Sivori, S. et al. Human NK cells: surface receptors, inhibitory checkpoints, and translational applications. Cell. Mol. Immunol. 16, 430-441 (2019).

5. Xu, X. et al. Association of germline variants in natural killer cells with tumor immune microenvironment subtypes, tumor-infiltrating lymphocytes, immunotherapy response, clinical outcomes, and cancer risk. JAMA Netw. Open 2, e199292 (2019).

6. Wu, Z. et al. Prognostic impact of adenosine receptor 2 (A2aR) and programmed cell death ligand 1 (PD-L1) expression in colorectal cancer. Biomed. Res. Int. 2019, 8014627 (2019).

7. Adachi, K. \& Tamada, K. Immune checkpoint blockade opens an avenue of cancer immunotherapy with a potent clinical efficacy. Cancer Sci. 106, 945-950 (2015).

8. Bern, M. D. et al. Immunoreceptor tyrosine-based inhibitory motif-dependent functions of an MHC class I-specific NK cell receptor. Proc. Natl Acad. Sci. USA 114, E8440-E8447 (2017).

9. Solomon, B. L. \& Garrido-Laguna, I. TIGIT: a novel immunotherapy target moving from bench to bedside. Cancer Immunol. Immunother. 67, 1659-1667 (2018).

10. Li, K. \& Tian, H. Development of small-molecule immune checkpoint inhibitors of PD-1/PD-L1 as a new therapeutic strategy for tumour immunotherapy. J. Drug Target. 27, 244-256 (2019).

11. Whelan, S. et al. PVRIG and PVRL2 are induced in cancer and inhibit CD8 ${ }^{+}$T-cell $^{-}$ function. Cancer Immunol. Res. 7, 257-268 (2019).

12. Gurjao, C. et al. Intrinsic resistance to immune checkpoint blockade in a mismatch repair-deficient colorectal cancer. Cancer Immunol. Res. 7, 1230-1236 (2019)

13. Tu, L. et al. Assessment of the expression of the immune checkpoint molecules PD-1, CTLA4, TIM-3 and LAG-3 across different cancers in relation to treatment response, tumor-infiltrating immune cells and survival. Int. J. Cancer 147, 423-439 (2020)

14. Souza-Fonseca-Guimaraes, F., Cursons, J. \& Huntington, N. D. The emergence of natural killer cells as a major target in cancer immunotherapy. Trends Immunol. 40, 142-158 (2019).

15. Zou, W. Mechanistic insights into cancer immunity and immunotherapy. Cell. Mol. Immunol. 15, 419-420 (2018).

16. André, $\mathrm{P}$. et al. Anti-NKG2A mAb is a checkpoint inhibitor that promotes antitumor immunity by unleashing both T and NK Cells. Cell 175, 1731-1743 (2018).

17. Carotta, S. Targeting NK cells for anticancer immunotherapy: clinical and preclinical approaches. Front. Immunol. 7, 152 (2016).

18. Genßler, S. et al. Dual targeting of glioblastoma with chimeric antigen receptorengineered natural killer cells overcomes heterogeneity of target antigen expression and enhances antitumor activity and survival. Oncoimmunology 5, e1119354 (2016)

19. Blake, S. J., Dougall, W. C., Miles, J. J., Teng, M. W. \& Smyth, M. J. Molecular pathways: targeting CD96 and TIGIT for cancer immunotherapy. Clin. Cancer Res. 22, 5183-5188 (2016).

20. Chen, Y. et al. Human fused NKG2D-IL-15 protein controls xenografted human gastric cancer through the recruitment and activation of NK cells. Cell. Mol. Immunol. 14, 293-307 (2017).
21. Nowak, J. et al. Role of donor activating KIR-HLA ligand-mediated NK cell education status in control of malignancy in hematopoietic cell transplant recipients. Biol. Blood Marrow Transplant. 21, 829-839 (2015).

22. van der Ploeg, $\mathrm{K}$. et al. Modulation of human leukocyte antigen-C by human cytomegalovirus stimulates KIR2DS1 recognition by natural killer cells. Front. Immunol. 8, 298 (2017).

23. Balsamo, M. et al. Hypoxia downregulates the expression of activating receptors involved in NK-cell-mediated target cell killing without affecting ADCC. Eur. J. Immunol. 43, 2756-2764 (2013).

24. Sun, H. \& Sun, C. The rise of NK cell checkpoints as promising therapeutic targets in cancer immunotherapy. Front. Immunol. 10, 2354 (2019).

25. Romagne, F. et al. Preclinical characterization of 1-7F9, a novel human anti-KIR receptor therapeutic antibody that augments natural killer-mediated killing of tumor cells. Blood 114, 2667-2677 (2009).

26. Khan, M., Arooj, S. \& Wang, H. NK cell-based immune checkpoint inhibition. Front. Immunol. 11, 167 (2020).

27. Riley, R. S., June, C. H., Langer, R. \& Mitchell, M. J. Delivery technologies for cancer immunotherapy. Nat. Rev. Drug Discov. 18, 175-196 (2019).

28. Sandoval-Borrego, D. et al. Overexpression of CD158 and NKG2A inhibitory receptors and underexpression of NKG2D and NKp46 activating receptors on NK cells in acute myeloid leukemia. Arch. Med. Res. 47, 55-64 (2016).

29. Mingari, M. C., Pietra, G. \& Moretta, L. Immune checkpoint inhibitors: anti-NKG2A antibodies on board. Trends Immunol. 40, 83-85 (2019).

30. Market, M., Baxter, K. E., Angka, L., Kennedy, M. A. \& Auer, R. C. The potential for cancer immunotherapy in targeting surgery-induced natural killer cell dysfunction. Cancers 11, 2 (2018).

31. Guillerey, C. et al. Immunosurveillance and therapy of multiple myeloma are CD226 dependent. J. Clin. Invest. 125, 2077-2089 (2015).

32. Parameswaran, R. et al. Repression of GSK3 restores NK cell cytotoxicity in AML patients. Nat Commun 7, 11154 (2016).

33. Delconte, R. B. et al. The helix-loop-helix protein ID2 governs NK cell fate by tuning their sensitivity to interleukin-15. Immunity 44, 103-115 (2016).

34. Blake, S. J. et al. Suppression of metastases using a new lymphocyte checkpoint target for cancer immunotherapy. Cancer Discov. 6, 446-459 (2016).

35. $\mathrm{Ma}, \mathrm{S}$. R. et al. Blockade of adenosine $\mathrm{A} 2 \mathrm{~A}$ receptor enhances $\mathrm{CD} 8^{+} \mathrm{T}$ cells response and decreases regulatory $T$ cells in head and neck squamous cell carcinoma. Mol. Cancer 16, 99 (2017).

36. Kamada, T. et al. PD- $1^{+}$regulatory T cells amplified by PD-1 blockade promote hyperprogression of cancer. Proc. Natl Acad. Sci. USA 116, 9999-10008 (2019).

37. Qin, S. et al. Novel immune checkpoint targets: moving beyond PD-1 and CTLA4. Mol. Cancer 18, 155 (2019).

38. Lanuza, P. M. et al. Activated human primary NK cells efficiently kill colorectal cancer cells in 3D spheroid cultures irrespectively of the level of PD-L1 expression. Oncoimmunology 7, e1395123 (2018).

39. Frazao, A. et al. NKG2D/NKG2-ligand pathway offers new opportunities in cancer treatment. Front. Immunol. 10, 661 (2019).

40. Bauman, Y. et al. Downregulation of the stress-induced ligand ULBP1 following SV40 infection confers viral evasion from NK cell cytotoxicity. Oncotarget 7, 15369-15381 (2016).

41. Ruggeri, L. et al. Effects of anti-NKG2A antibody administration on leukemia and normal hematopoietic cells. Haematologica 101, 626-633 (2016).

42. Darvin, P., Toor, S. M., Sasidharan Nair, V. \& Elkord, E. Immune checkpoint inhibitors: recent progress and potential biomarkers. Exp. Mol. Med. 50, 1-11 (2018).

43. Kamiya, T., Seow, S. V., Wong, D., Robinson, M. \& Campana, D. Blocking expression of inhibitory receptor NKG2A overcomes tumor resistance to NK cells. J. Clin. Invest. 129, 2094-2106 (2019).

44. Jiang, Y., Chen, M., Nie, H. \& Yuan, Y. PD-1 and PD-L1 in cancer immunotherapy: clinical implications and future considerations. Hum. Vaccin. Immunother. 15, 1111-1122 (2019).

45. He, W. et al. CD155T/TIGIT signaling regulates $\mathrm{CD}^{+}{ }^{+}$T-cell metabolism and promotes tumor progression in human gastric cancer. Cancer Res. 77, 6375-6388 (2017).

46. Sanchez-Correa, B. et al. Modulation of NK cells with checkpoint inhibitors in the context of cancer immunotherapy. Cancer Immunol. Immunother. 68, 861-870 (2019).

47. Zhou, G. et al. Reduction of immunosuppressive tumor microenvironment in cholangiocarcinoma by ex vivo targeting immune checkpoint molecules. $J$. Hepatol. 71, 753-762 (2019).

48. Feng, M. et al. Phagocytosis checkpoints as new targets for cancer immunotherapy. Nat. Rev. Cancer 19, 568-586 (2019).

49. Yang, E., Singh, B. K., Paustian, A. M. \& Kambayashi, T. Diacylglycerol Kinase $\zeta$ is a target to enhance NK cell function. J. Immunol. 197, 934-941 (2016).

50. Lanuza, P. M. et al. Recalling the biological significance of immune checkpoints on NK cells: a chance to overcome LAG3, PD1, and CTLA4 inhibitory pathways by adoptive NK cell transfer? Front. Immunol. 10, 3010 (2019). 
51. Meza Guzman, L. G., Keating, N. \& Nicholson, S. E. Natural killer cells: tumor surveillance and signaling. Cancers 12, 952 (2020).

52. Teratake, Y. et al. Development of a protein-based system for transient epigenetic repression of immune checkpoint molecule and enhancement of antitumour activity of natural killer cells. Br. J. Cancer 122, 823-834 (2020).

53. Miller, J. S. \& Lanier, L. L. Natural killer cells in cancer immunotherapy. Ann. Rev. Cancer Biol. 3, 77-103 (2019).

54. Fang, F., Xiao, W. \& Tian, Z. Challenges of NK cell-based immunotherapy in the new era. Front. Med. 12, 440-450 (2018).

55. Battella, S., Cox, M. C., Santoni, A. \& Palmieri, G. Natural killer (NK) cells and antitumor therapeutic mAb: unexplored interactions. J. Leukoc. Biol. 99, 87-96 (2016).

56. Xu-Monette, Z. Y. et al. Immune profiling and quantitative analysis decipher the clinical role of immune-checkpoint expression in the tumor immune microenvironment of DLBCL. Cancer Immunol. Res. 7, 644-657 (2019).

57. Cantoni, C. et al. NK cells, tumor cell transition, and tumor progression in solid malignancies: new hints for NK-based immunotherapy? J. Immunol. Res. 2016, 4684268 (2016).

58. Nabatanzi, R., Cose, S., Joloba, M., Jones, S. R. \& Nakanjako, D. Effects of HIV infection and ART on phenotype and function of circulating monocytes, natural killer, and innate lymphoid cells. AIDS Res. Ther. 15, 7 (2018).

59. Hood, S. P. et al. Phenotype and function of activated natural killer cells from patients with prostate cancer: patient-dependent responses to priming and IL-2 activation. Front. Immunol. 9, 3169 (2018).

60. Bjorkstrom, N. K. et al. Expression patterns of NKG2A, KIR, and CD57 define a process of $\mathrm{CD} 56^{\mathrm{dim}}$ NK-cell differentiation uncoupled from NK-cell education. Blood 116, 3853-3864 (2010).

61. Chiossone, L., Vienne, M., Kerdiles, Y. M. \& Vivier, E. Natural killer cell immunotherapies against cancer: checkpoint inhibitors and more. Semin. Immunol. 31, 55-63 (2017).

62. Munari, E. et al. PD-L1 expression heterogeneity in non-small cell lung cancer: defining criteria for harmonization between biopsy specimens and whole sections. J. Thorac. Oncol. 13, 1113-1120 (2018).

63. Chretien, A. S. et al. Cancer-induced alterations of NK-mediated target recognition: current and investigational pharmacological strategies aiming at restoring NK-mediated anti-tumor activity. Front. Immunol. 5, 122 (2014).

64. Huang, R. et al. Loss of Fas expression and high expression of HLA-E promoting the immune escape of early colorectal cancer cells. Oncol. Lett. 13, 3379-3386 (2017).

65. Sun, H. et al. Human CD96 correlates to natural killer cell exhaustion and predicts the prognosis of human hepatocellular Carcinoma. Hepatology 70 , 168-183 (2019).

66. Yoon, S. R., Kim, T. D. \& Choi, I. Understanding of molecular mechanisms in natural killer cell therapy. Exp. Mol. Med. 47, e141 (2015).

67. Konjević, G., Vuletić, A. \& Mirjačić Martinović, K. Natural killer cell receptors: alterations and therapeutic targeting in malignancies. Immunol. Res. 64, 25-35 (2016).

68. Thiruchelvam-Kyle, L. et al. The activating human NK cell receptor KIR2DS2 recognizes a $\beta_{2}$-microglobulin-independent ligand on cancer cells. J. Immunol. 198, 2556-2567 (2017).

69. Chaganty, B. K. et al. Trastuzumab upregulates expression of HLA-ABC and T cell costimulatory molecules through engagement of natural killer cells and stimulation of IFNY secretion. Oncoimmunology 5, e1100790 (2016).

70. Torphy, R. J., Schulick, R. D. \& Zhu, Y. Newly emerging immune checkpoints: promises for future cancer therapy. Int. J. Mol. Sci. 18, 2642 (2017).

71. Xu, L. et al. Increased Tim-3 expression in peripheral NK cells predicts a poore prognosis and Tim-3 blockade improves NK cell-mediated cytotoxicity in human lung adenocarcinoma. Int. Immunopharmacol. 29, 635-641 (2015).

72. Pesce, S. et al. Identification of a subset of human natural killer cells expressing high levels of programmed death 1: a phenotypic and functional characterization. J. Allergy Clin. Immunol. 139, 335-346.e333 (2017).

73. Tumino, N. et al. Presence of innate lymphoid cells in pleural effusions of primary and metastatic tumors: functional analysis and expression of PD-1 receptor. Int. J. Cancer 145, 1660-1668 (2019).

74. Muntasell, A. et al. Targeting NK-cell checkpoints for cancer immunotherapy. Curr. Opin. Immunol. 45, 73-81 (2017).

75. Wang, Q., Gao, J. \& Wu, X. Pseudoprogression and hyperprogression after checkpoint blockade. Int. Immunopharmacol. 58, 125-135 (2018).

76. Paul, S. \& Lal, G. The molecular mechanism of natural killer cells function and its importance in cancer immunotherapy. Front. Immunol. 8, 1124 (2017).

77. Vari, F. et al. Immune evasion via PD-1/PD-L1 on NK cells and monocyte/macrophages is more prominent in Hodgkin lymphoma than DLBCL. Blood 131, 1809-1819 (2018).

78. Jung, H. I. et al. Overexpression of PD-L1 and PD-L2 is associated with poor prognosis in patients with hepatocellular carcinoma. Cancer Res. Treat. 49, 246-254 (2017).
79. Peled, M. et al. Affinity purification mass spectrometry analysis of PD-1 uncovers SAP as a new checkpoint inhibitor. Proc. Natl Acad. Sci. USA 115, E468-e477 (2018).

80. Herndler-Brandstetter, D. et al. Humanized mouse model supports development, function, and tissue residency of human natural killer cells. Proc. Nat Acad. Sci. USA 114, E9626-e9634 (2017).

81. Hsu, J. et al. Contribution of NK cells to immunotherapy mediated by PD-1/PDL1 blockade. J. Clin. Invest. 128, 4654-4668 (2018).

82. Nishimura, Y. et al. Histidine-rich glycoprotein augments natural killer cell function by modulating PD-1 expression via CLEC-1B. Pharmacol. Res. Perspect. 7, e00481 (2019).

83. Jeffery, H. C. et al. Changes in natural killer cells and exhausted memory regulatory T Cells with corticosteroid therapy in acute autoimmune hepatitis. Hepatol. Commun. 2, 421-436 (2018).

84. Delconte, R. B. et al. CIS is a potent checkpoint in NK cell-mediated tumor immunity. Nat. Immunol. 17, 816-824 (2016).

85. Beldi-Ferchiou, A. et al. PD-1 mediates functional exhaustion of activated NK cells in patients with Kaposi sarcoma. Oncotarget 7, 72961-72977 (2016).

86. Tabellini, G. et al. Primitive neuroectodermal tumor in an ovarian cystic teratoma: natural killer and neuroblastoma cell analysis. Case Rep. Oncol. 7, 70-78 (2014).

87. Liu, Y. et al. Increased expression of programmed cell death protein 1 on NK cells inhibits NK-cell-mediated anti-tumor function and indicates poor prognosis in digestive cancers. Oncogene 36, 6143-6153 (2017).

88. Xie, S., Chen, J., Zhang, M. \& Wu, Z. Allogenic natural killer cell immunotherapy of sizeable ovarian cancer: a case report. Mol. Clin. Oncol. 6, 903-906 (2017).

89. Shitara, K. \& Nishikawa, H. Regulatory T cells: a potential target in cancer immunotherapy. Ann. NY Acad. Sci. 1417, 104-115 (2018).

90. Stein, N., Tsukerman, P. \& Mandelboim, O. The paired receptors TIGIT and DNAM-1 as targets for therapeutic antibodies. Hum. Antibodies 25, 111-119 (2017).

91. Mahaweni, N. M., Ehlers, F. A. I., Bos, G. M. J. \& Wieten, L. Tuning natural killer cell anti-multiple myeloma reactivity by targeting inhibitory signaling via KIR and NKG2A. Front. Immunol. 9, 2848 (2018).

92. Li, M. et al. T-cell immunoglobulin and ITIM domain (TIGIT) receptor/poliovirus receptor (PVR) ligand engagement suppresses interferon- $\gamma$ production of natural killer cells via $\beta$-arrestin 2-mediated negative signaling. J. Biol. Chem. 289, 17647-17657 (2014)

93. Hong, X., Wang, X., Wang, T. \& Zhang, X. Correlation of T cell immunoglobulin and ITIM domain (TIGIT) and programmed death 1 (PD-1) with clinicopathological characteristics of renal cell carcinoma may indicate potential targets for treatment. Med. Sci. Monit. 24, 6861-6872 (2018).

94. Cho, Y. H. et al. Natural killer cells as a potential biomarker for predicting immunotherapy efficacy in patients with non-small cell lung cancer. Target. Oncol. 15, 241-247 (2020).

95. Wagner, J. et al. A two-phase expansion protocol combining interleukin (IL)-15 and IL-21 improves natural killer cell proliferation and cytotoxicity against rhabdomyosarcoma. Front. Immunol. 8, 676 (2017).

96. Morvan, M. G. \& Lanier, L. L. NK cells and cancer: you can teach innate cells new tricks. Nat. Rev. Cancer 16, 7-19 (2016).

97. Zhang, Q. et al. Blockade of the checkpoint receptor TIGIT prevents NK cell exhaustion and elicits potent anti-tumor immunity. Nat. Immunol. 19, 723-732 (2018).

98. Zhang, B. et al. Immunoreceptor TIGIT inhibits the cytotoxicity of human cytokine-induced killer cells by interacting with CD155. Cancer Immunol. Immunother. 65, 305-314 (2016).

99. Kurtulus, S. et al. TIGIT predominantly regulates the immune response via regulatory T cells. J. Clin. Invest. 125, 4053-4062 (2015).

100. Inozume, T. et al. Melanoma cells control antimelanoma CTL responses via interaction between TIGIT and CD155 in the effector phase. J. Invest. Dermatol. 136, 255-263 (2016).

101. Kim, N. et al. Natural killer cells as a promising therapeutic target for cancer immunotherapy. Arch. Pharm. Res. 42, 591-606 (2019).

102. Jong, A. Y. et al. Large-scale isolation and cytotoxicity of extracellular vesicles derived from activated human natural killer cells. J. Extracell. Vesicles 6, 1294368 (2017).

103. Sun, Y. et al. Combined evaluation of the expression status of CD155 and TIGIT plays an important role in the prognosis of LUAD (lung adenocarcinoma). Int. Immunopharmacol. 80, 106198 (2020).

104. Kim, N. \& Kim, H. S. Targeting checkpoint receptors and molecules for therapeutic modulation of natural killer cells. Front. Immunol. 9, 2041 (2018).

105. Liu, S. et al. Recruitment of Grb2 and SHIP1 by the ITT-like motif of TIGIT suppresses granule polarization and cytotoxicity of NK cells. Cell Death Differ. 20, 456-464 (2013)

106. Manieri, N. A., Chiang, E. Y. \& Grogan, J. L. TIGIT: a key inhibitor of the cancer immunity cycle. Trends Immunol. 38, 20-28 (2017). 
107. Boyerinas, B. et al. Antibody-dependent cellular cytotoxicity activity of a novel anti-PD-L1 antibody avelumab (MSB0010718C) on human tumor cells. Cancer Immunol. Res. 3, 1148-1157 (2015).

108. Sarhan, D. et al. Adaptive NK cells resist regulatory T-cell suppression driven by IL37. Cancer Immunol. Res. 6, 766-IL775 (2018).

109. Ma, B. et al. Use of aspirin in the prevention of colorectal cancer through TIGITCD155 pathway. J. Cell. Mol. Med. 23, 4514-4522 (2019).

110. Meckawy, G. R. et al. Natural killer NKG2A and NKG2D in patients with colorectal cancer. J. Gastrointest. Oncol. 10, 218-225 (2019).

111. Chan, C. J. et al. The receptors CD96 and CD226 oppose each other in the regulation of natural killer cell functions. Nat. Immunol. 15, 431-438 (2014).

112. Bernhardt, G. TACTILE becomes tangible: CD96 discloses its inhibitory peculiarities. Nat. Immunol. 15, 406-408 (2014).

113. Carlsten, M. \& Childs, R. W. Genetic manipulation of NK cells for cancer immunotherapy: techniques and clinical implications. Front. Immunol. 6, 266 (2015).

114. Rezvani, K., Rouce, R., Liu, E. \& Shpall, E. Engineering natural killer cells for cancer immunotherapy. Mol. Ther. 25, 1769-1781 (2017).

115. Hu, W., Wang, G., Huang, D., Sui, M. \& Xu, Y. Cancer immunotherapy based on natural killer cells: current progress and new opportunities. Front. Immunol. 10, 1205 (2019).

116. Deuss, F. A., Gully, B. S., Rossjohn, J. \& Berry, R. Recognition of nectin-2 by the natural killer cell receptor T cell immunoglobulin and ITIM domain (TIGIT). J. Biol. Chem. 292, 11413-11422 (2017).

117. Holmes, V. M. et al. Interaction between nectin-1 and the human natural killer cell receptor CD96. PLoS One 14, e0212443 (2019).

118. Deuss, F. A., Watson, G. M., Fu, Z., Rossjohn, J. \& Berry, R. Structural basis for CD96 immune receptor recognition of nectin-like protein-5, CD155. Structure 27, 219-228.e213 (2019)

119. Hodgins, J. J., Khan, S. T., Park, M. M., Auer, R. C. \& Ardolino, M. Killers 2.0: NK cell therapies at the forefront of cancer control. J. Clin. Invest. 129, 3499-3510 (2019).

120. Roman Aguilera, A. et al. CD96 targeted antibodies need not block CD96-CD155 interactions to promote NK cell anti-metastatic activity. Oncoimmunology $\mathbf{7}$, e1424677 (2018)

121. Vitale, M., Cantoni, C., Pietra, G., Mingari, M. C. \& Moretta, L. Effect of tumor cells and tumor microenvironment on NK-cell function. Eur. J. Immunol. 44, 1582-1592 (2014).

122. Wang, Z. et al. The clinical significance of abnormal Tim-3 expression on NK cells from patients with gastric cancer. Immunol. Invest. 44, 578-589 (2015).

123. Komita, H. et al. Expression of immune checkpoint molecules of T cell immunoglobulin and mucin protein 3/galectin-9 for NK cell suppression in human gastrointestinal stromal tumors. Oncol. Rep. 34, 2099-2105 (2015).

124. Cekic, C., Day, Y. J., Sag, D. \& Linden, J. Myeloid expression of adenosine A2A receptor suppresses $T$ and NK cell responses in the solid tumor microenvironment. Cancer Res. 74, 7250-7259 (2014).

125. Meggyes, M. et al. Peripheral blood TIM-3 positive NK and CD8+ T cells throughout pregnancy: TIM-3/galectin-9 interaction and its possible role during pregnancy. PLoS ONE 9, e92371 (2014).

126. Kikushige, Y. et al. A TIM-3/Gal-9 autocrine stimulatory loop drives self-renewal of human myeloid leukemia stem cells and leukemic progression. Cell Stem Cell 17, 341-352 (2015).

127. Vega-Carrascal, I. et al. Dysregulation of TIM-3-galectin-9 pathway in the cystic fibrosis airways. J. Immunol. 186, 2897-2909 (2011).

128. Van Audenaerde, J. R. M. et al. Interleukin-15 stimulates natural killer cellmediated killing of both human pancreatic cancer and stellate cells. Oncotarget 8, 56968-56979 (2017)

129. So, E. C. et al. NK cell expression of Tim-3: first impressions matter. Immunobiology 224, 362-370 (2019).

130. Hadadi, L. et al. Dysregulated expression of Tim-3 and NKp30 receptors on NK cells of patients with chronic lymphocytic leukemia. Oncol Res Treat 42, 202-208 (2019).

131. $\mathrm{Lu}$, X. et al. Tumor antigen-specific $\mathrm{CD} 8^{+} \mathrm{T}$ cells are negatively regulated by PD1 and Tim-3 in human gastric cancer. Cell. Immunol. 313, 43-51 (2017).

132. Gorman, J. V. \& Colgan, J. D. Regulation of T cell responses by the receptor molecule Tim-3. Immunol. Res. 59, 56-65 (2014).

133. Komohara, Y. et al. The coordinated actions of TIM-3 on cancer and myeloid cells in the regulation of tumorigenicity and clinical prognosis in clear cell renal cell carcinomas. Cancer Immunol. Res. 3, 999-1007 (2015).

134. Shayan, G. et al. Adaptive resistance to anti-PD1 therapy by Tim-3 upregulation is mediated by the PI3K-Akt pathway in head and neck cancer. Oncoimmunology 6, e1261779 (2017).

135. Freud, A. G., Yu, J. \& Caligiuri, M. A. Human natural killer cell development in secondary lymphoid tissues. Semin. Immunol. 26, 132-137 (2014).

136. Li, Z., Liu, X., Guo, R. \& Wang, P. TIM-3 plays a more important role than PD-1 in the functional impairments of cytotoxic $T$ cells of malignant Schwannomas. Tumour Biol. 39, 1010428317698352 (2017).
137. Sanmamed, M. F. \& Chen, L. A paradigm shift in cancer immunotherapy: from enhancement to normalization. Cell 175, 313-326 (2018).

138. He, Y. et al. TIM-3, a promising target for cancer immunotherapy. Onco Targets Ther. 11, 7005-7009 (2018)

139. Ndhlovu, L. C. et al. Tim-3 marks human natural killer cell maturation and suppresses cell-mediated cytotoxicity. Blood 119, 3734-3743 (2012).

140. Wang, Y. et al. Preoperative Tim-3 expression on peripheral NK cells is correlated with pathologic TNM staging in colorectal cancer. Mol. Med. Rep. 15, 3810-3818 (2017).

141. Yang, M. et al. T-cell immunoglobulin mucin-3 expression in bladder urothelial carcinoma: clinicopathologic correlations and association with survival. J. Surg. Oncol. 112, 430-435 (2015).

142. Zhang, Y. \& Schmidt-Wolf, I. G. H. Ten-year update of the international registry on cytokine-induced killer cells in cancer immunotherapy. J. Cell. Physiol. 235, 9291-9303 (2020).

143. Ju, Y. et al. T cell immunoglobulin- and mucin-domain-containing molecule-3 (Tim-3) mediates natural killer cell suppression in chronic hepatitis B. J. Hepatol. 52, 322-329 (2010).

144. Huang, Y. H. et al. CEACAM1 regulates TIM-3-mediated tolerance and exhaustion. Nature 517, 386-390 (2015)

145. Melero, I., Rouzaut, A., Motz, G. T. \& Coukos, G. T-cell and NK-cell infiltration into solid tumors: a key limiting factor for efficacious cancer immunotherapy. Cancer Discov. 4, 522-526 (2014).

146. Zhang, X. et al. Increased Tim-3 expression on TILs during treatment with the Anchored GM-CSF vaccine and anti-PD-1 antibodies is inversely correlated with response in prostate cancer. J. Cancer 11, 648-656 (2020).

147. Sabatos-Peyton, C. A. et al. Blockade of Tim-3 binding to phosphatidylserine and CEACAM1 is a shared feature of anti-Tim-3 antibodies that have functional efficacy. Oncoimmunology 7, e1385690 (2018).

148. van de Weyer, P. S. et al. A highly conserved tyrosine of Tim-3 is phosphorylated upon stimulation by its ligand galectin-9. Biochem. Biophys. Res. Commun. 351, 571-576 (2006).

149. Lee, J. et al. Phosphotyrosine-dependent coupling of Tim-3 to T-cell receptor signaling pathways. Mol. Cell. Biol. 31, 3963-3974 (2011).

150. Rangachari, M. et al. Bat3 promotes $T$ cell responses and autoimmunity by repressing Tim-3-mediated cell death and exhaustion. Nat. Med. 18, 1394-1400 (2012).

151. Ji, J. et al. Long non-coding RNA Lnc-Tim3 exacerbates CD8 T cell exhaustion via binding to Tim-3 and inducing nuclear translocation of Bat3 in HCC. Cell Death Dis. 9, 478 (2018).

152. da Silva, I. P. et al. Reversal of NK-cell exhaustion in advanced melanoma by Tim3 blockade. Cancer Immunol. Res. 2, 410-422 (2014).

153. Du, W. et al. TIM-3 as a target for cancer immunotherapy and mechanisms of action. Int. J. Mol. Sci. 18, 645 (2017).

154. Tallerico, R. et al. IL-15, TIM-3 and NK cells subsets predict responsiveness to anti-CTLA-4 treatment in melanoma patients. Oncoimmunology 6, e1261242 (2017).

155. Schönfeld, K. et al. Selective inhibition of tumor growth by clonal NK cells expressing an ErbB2/HER2-specific chimeric antigen receptor. Mol. Ther. 23, 330-338 (2015).

156. Anderson, A. C., Joller, N. \& Kuchroo, V. K. Lag-3, Tim-3, and TIGIT: co-inhibitory receptors with specialized functions in immune regulation. Immunity $\mathbf{4 4}$, 989-1004 (2016).

157. van de Donk, N. W. et al. Clinical efficacy and management of monoclonal antibodies targeting CD38 and SLAMF7 in multiple myeloma. Blood 127, 681-695 (2016).

158. Murter, B. et al. Mouse PVRIG has $\mathrm{CD}^{+} \mathrm{T}$ cell-specific coinhibitory functions and dampens antitumor immunity. Cancer Immunol. Res. 7, 244-256 (2019).

159. Zhu, Y. et al. Identification of CD112R as a novel checkpoint for human T cells. J. Exp. Med. 213, 167-176 (2016).

160. Xu, F. et al. Blockade of CD112R and TIGIT signaling sensitizes human natural killer cell functions. Cancer Immunol. Immunother. 66, 1367-1375 (2017).

161. Krasnova, Y., Putz, E. M., Smyth, M. J. \& Souza-Fonseca-Guimaraes, F. Bench to bedside: NK cells and control of metastasis. Clin. Immunol. 177, 50-59 (2017).

162. Julia, E. P., Amante, A., Pampena, M. B., Mordoh, J. \& Levy, E. M. Avelumab, an IgG1 anti-PD-L1 immune checkpoint inhibitor, triggers NK cell-mediated cytotoxicity and cytokine production against triple negative breast cancer cells. Front. Immunol. 9, 2140 (2018).

163. Capone, $M$. et al. Frequency of circulating CD8 $+C D 73+T$ cells is associated with survival in nivolumab-treated melanoma patients. J. Transl. Med. 18, 121 (2020).

164. Lupo, K. B. \& Matosevic, S. Natural killer cells as allogeneic effectors in adoptive cancer immunotherapy. Cancers 11, 769 (2019).

165. Molgora, M., Supino, D., Mantovani, A. \& Garlanda, C. Tuning inflammation and immunity by the negative regulators IL-1R2 and IL-1R8. Immunol. Rev. 281, 233-247 (2018). 
166. Guillerey, C. et al. Toll-like receptor 3 regulates NK cell responses to cytokines and controls experimental metastasis. Oncoimmunology 4, e1027468 (2015).

167. Molgora, M. et al. IL-1R8 is a checkpoint in NK cells regulating anti-tumour and anti-viral activity. Nature 551, 110-114 (2017).

168. Seo, H. et al. IL-21-mediated reversal of NK cell exhaustion facilitates anti-tumour immunity in MHC class I-deficient tumours. Nat. Commun. 8, 15776 (2017).

169. Chang, Y. H. et al. A chimeric receptor with NKG2D specificity enhances natural killer cell activation and killing of tumor cells. Cancer Res. 73, 1777-1786 (2013).

170. Romanski, A. et al. CD19-CAR engineered NK-92 cells are sufficient to overcome NK cell resistance in B-cell malignancies. J. Cell. Mol. Med. 20, 1287-1294 (2016).

171. Oelsner, S. et al. Continuously expanding CAR NK-92 cells display selective cytotoxicity against B-cell leukemia and lymphoma. Cytotherapy 19, 235-249 (2017).

172. Ao, X. et al. Anti-aFR CAR-engineered NK-92 cells display potent cytotoxicity against aFR-positive ovarian cancer. J. Immunother. 42, 284-296 (2019).

173. Montagner, I. M. et al. Anti-PSMA CAR-engineered NK-92 cells: an off-the-shelf cell therapy for prostate cancer. Cells 9, 1382 (2020).

174. Ruffo, E., Wu, R. C., Bruno, T. C., Workman, C. J. \& Vignali, D. A. A. Lymphocyteactivation gene 3 (LAG3): the next immune checkpoint receptor. Semin. Immunol. 42, 101305 (2019).

175. Xu, F. et al. LSECtin expressed on melanoma cells promotes tumor progression by inhibiting antitumor T-cell responses. Cancer Res. 74, 3418-3428 (2014).

176. Andrews, L. P., Marciscano, A. E., Drake, C. G. \& Vignali, D. A. LAG3 (CD223) as a cancer immunotherapy target. Immunol. Rev. 276, 80-96 (2017).

177. Williams, P. et al. The distribution of T-cell subsets and the expression of immune checkpoint receptors and ligands in patients with newly diagnosed and relapsed acute myeloid leukemia. Cancer 125, 1470-1481 (2019).

178. Gandhi, M. K. et al. Expression of LAG-3 by tumor-infiltrating lymphocytes is coincident with the suppression of latent membrane antigen-specific CD8 ${ }^{+}$ T-cell function in Hodgkin lymphoma patients. Blood 108, 2280-2289 (2006).

179. Shapiro, M. et al. Lymphocyte activation gene 3 : a novel therapeutic target in chronic lymphocytic leukemia. Haematologica 102, 874-882 (2017).

180. Workman, C. J., Dugger, K. J. \& Vignali, D. A. Cutting edge: molecular analysis of the negative regulatory function of lymphocyte activation gene-3. J. Immunol. 169, 5392-5395 (2002).

181. Wang, J. et al. Fibrinogen-like protein 1 is a major immune inhibitory ligand of LAG-3. Cell 176, 334-347 e312 (2019).

182. Burton, B. R. et al. Sequential transcriptional changes dictate safe and effective antigen-specific immunotherapy. Nat. Commun. 5, 4741 (2014).

183. Catakovic, K., Klieser, E., Neureiter, D. \& Geisberger, R. T cell exhaustion: from pathophysiological basics to tumor immunotherapy. Cell Commun. Signal. 15, 1 (2017).

184. Marçais, A. et al. The metabolic checkpoint kinase mTOR is essential for IL-15 signaling during the development and activation of NK cells. Nat. Immunol. 15, 749-757 (2014).

185. Marhelava, K., Pilch, Z., Bajor, M., Graczyk-Jarzynka, A. \& Zagozdzon, R. Targeting negative and positive immune checkpoints with monoclonal antibodies in therapy of cancer. Cancers 11, 1756 (2019).

186. Das, M., Zhu, C. \& Kuchroo, V. K. Tim-3 and its role in regulating anti-tumor immunity. Immunol. Rev. 276, 97-111 (2017).

187. Meyer, C. E. et al. Expression of the inhibitory receptor NKG2A correlates with increased liver and splenic NK cell response to activating receptor engagement. Immun. Inflamm. Dis. 5, 177-189 (2017).

188. Ben-Shmuel, A., Biber, G., Sabag, B. \& Barda-Saad, M. Modulation of the intracellular inhibitory checkpoint SHP-1 enhances the antitumor activity of engineered NK cells. Cell. Mol. Immunol. https://doi.org/10.1038/s41423-020-0443-6 (2020).

189. Kumar, S. Natural killer cell cytotoxicity and its regulation by inhibitory receptors. Immunology 154, 383-393 (2018)

190. Lowry, L. E. \& Zehring, W. A. Potentiation of natural killer cells for cancer immunotherapy: a review of literature. Front. Immunol. 8, 1061 (2017).

191. Mukherjee, N. et al. Intratumoral CD56 ${ }^{\text {bright }}$ natural killer cells are associated with improved survival in bladder cancer. Oncotarget 9, 36492-36502 (2018).

192. Chang, W. C. et al. Regulatory T cells suppress natural killer cell immunity in patients with human cervical carcinoma. Int. J. Gynecol. Cancer 26, 156-162 (2016).

193. Sun, C. et al. High NKG2A expression contributes to NK cell exhaustion and predicts a poor prognosis of patients with liver cancer. Oncoimmunology 6, e1264562 (2017).

194. Böttcher, J. P. et al. NK cells stimulate recruitment of CDC1 into the tumor microenvironment promoting cancer immune control. Cell 172, 1022-1037. e1014 (2018).

195. Nabekura, T. et al. Cutting edge: NKG2D signaling enhances NK cell responses but alone is insufficient to drive expansion during mouse cytomegalovirus infection. J. Immunol. 199, 1567-1571 (2017).
196. Miyazato, K. \& Hayakawa, Y. Pharmacological targeting of natural killer cells for cancer immunotherapy. Cancer Sci. 111, 1869-1875 (2020).

197. Carlsten, M. et al. Checkpoint inhibition of KIR2D with the monoclonal antibody IPH2101 induces contraction and hyporesponsiveness of NK cells in patients with myeloma. Clin. Cancer Res. 22, 5211-5222 (2016).

198. Hargadon, K. M., Johnson, C. E. \& Williams, C. J. Immune checkpoint blockade therapy for cancer: An overview of FDA-approved immune checkpoint inhibitors. Int. Immunopharmacol. 62, 29-39 (2018).

199. Sanchez-Correa, B. et al. DNAM-1 and the TIGIT/PVRIG/TACTILE axis: novel immune checkpoints for natural killer cell-based cancer immunotherapy. Cancers 11, 877 (2019).

200. Bauer, S. et al. Activation of NK cells and T cells by NKG2D, a receptor for stress inducible MICA. Science 285, 727-729 (1999).

201. Yang, C., Li, Y., Yang, Y. \& Chen, Z. Overview of strategies to improve therapy against tumors using natural killer cell. J Immunol. Res. 2020, 8459496 (2020).

202. van Hall, T. et al. Monalizumab: inhibiting the novel immune checkpoint NKG2A. J. Immunother. Cancer 7, 263 (2019).

203. Hosomi, S., Grootjans, J., Huang, Y. H., Kaser, A. \& Blumberg, R. S. New insights into the regulation of natural-killer group 2 member D (NKG2D) and NKG2Dligands: endoplasmic reticulum stress and CEA-related cell adhesion molecule 1. Front. Immunol. 9, 1324 (2018).

204. McWilliams, E. M. et al. Therapeutic CD94/NKG2A blockade improves natural killer cell dysfunction in chronic lymphocytic leukemia. Oncoimmunology $\mathbf{5}$, e1226720 (2016).

205. Grossenbacher, S. K., Canter, R. J. \& Murphy, W. J. Natural killer cell immunotherapy to target stem-like tumor cells. J Immunother. Cancer 4, 19 (2016).

206. Ames, E. et al. NK cells preferentially target tumor cells with a cancer stem cell phenotype. J. Immunol. 195, 4010-4019 (2015).

207. Deng, W. et al. Antitumor immunity. A shed NKG2D ligand that promotes natural killer cell activation and tumor rejection. Science 348, 136-139 (2015).

208. Luo, Q. et al. Tumor-derived soluble MICA obstructs the NKG2D pathway to restrain NK cytotoxicity. Aging Dis. 11, 118-128 (2020).

209. Du, C. et al. MICA immune complex formed with alpha 3 domain-specific antibody activates human NK cells in a Fc-dependent manner. J. Immunother. Cancer 7, 207 (2019).

210. Wei, F. et al. BPIFB1 (LPLUNC1) inhibits radioresistance in nasopharyngeal carcinoma by inhibiting VTN expression. Cell Death Dis. 9, 432 (2018).

211. Pérez-Martínez, A. et al. A phase $1 / I I$ trial of interleukin-15-stimulated natural killer cell infusion after haplo-identical stem cell transplantation for pediatric refractory solid tumors. Cytotherapy 17, 1594-1603 (2015).

212. Kloess, S. et al. Triplebody mediates increased anti-leukemic reactivity of IL-2 activated donor natural killer (NK) cells and impairs viability of their CD33expressing NK subset. Front. Immunol. 8, 1100 (2017).

213. Romero, A. I., Thoren, F. B., Brune, M. \& Hellstrand, K. NKp46 and NKG2D receptor expression in NK cells with $\mathrm{CD} 56^{\mathrm{dim}}$ and $\mathrm{CD} 56^{\text {bright }}$ phenotype: regulation by histamine and reactive oxygen species. Br. J. Haematol. 132, 91-98 (2006).

214. Lee, J. C., Lee, K. M., Ahn, Y. O., Suh, B. \& Heo, D. S. A possible mechanism of impaired NK cytotoxicity in cancer patients: down-regulation of DAP10 by TGFbeta1. Tumori J. 97, 350-357 (2011).

215. Baragano Raneros, A. et al. Methylation of NKG2D ligands contributes to immune system evasion in acute myeloid leukemia. Genes Immun. 16, 71-82 (2015).

216. Shah, N. N. et al. Acute GVHD in patients receiving IL-15/4-1BBL activated NK cells following T-cell-depleted stem cell transplantation. Blood 125, 784-792 (2015).

217. Li, Y. \& Di Santo, J. P. Probing human NK cell biology using human immune system (HIS) mice. Curr. Top. Microbiol. Immunol. 395, 191-208 (2016).

218. Kwon, H. J., Kim, N. \& Kim, H. S. Molecular checkpoints controlling natural killer cell activation and their modulation for cancer immunotherapy. Exp. Mol. Med. 49, e311 (2017).

219. Bachanova, V. et al. Haploidentical natural killer cells induce remissions in nonHodgkin lymphoma patients with low levels of immune-suppressor cells. Cancer Immunol. Immunother. 67, 483-494 (2018).

220. He, Y. et al. The combination of anti-KIR monoclonal antibodies with anti-PD-1/ PD-L1 monoclonal antibodies could be a critical breakthrough in overcoming tumor immune escape in NSCLC. Drug Des. Devel. Ther. 12, 981-986 (2018).

221. Kandilarova, S. M. et al. The influence of HLA and KIR genes on malignant melanoma development and progression. Arch. Immunol. Ther. Exp. 64, 73-81 (2016).

222. Rautela, J et al. Molecular insight into targeting the NK cell immune response to cancer. Immunol. Cell Biol. 96, 477-484 (2018).

223. Levi-Schaffer, F. \& Mandelboim, O. Inhibitory and coactivating receptors recognising the same ligand: immune homeostasis exploited by pathogens and tumours. Trends Immunol. 39, 112-122 (2018).

224. Zamora, A. E., Grossenbacher, S. K., Aguilar, E. G. \& Murphy, W. J. Models to study NK cell biology and possible clinical application. Curr. Protoc. Immunol. 110, 14 37 11-14 3714 (2015). 
225. Mulrooney, T. J., Posch, P. E. \& Hurley, C. K. DAP12 impacts trafficking and surface stability of killer immunoglobulin-like receptors on natural killer cells. J. Leukoc. Biol. 94, 301-313 (2013).

226. Chambers, A. M. \& Matosevic, S. Immunometabolic dysfunction of natural killer cells mediated by the hypoxia-CD73 axis in solid tumors. Front. Mol. Biosci. 6, 60 (2019).

227. Sakamoto, N. et al. Phase I clinical trial of autologous NK cell therapy using novel expansion method in patients with advanced digestive cancer. J. Transl. Med. 13, 277 (2015)

228. Hilton, H. G. et al. The production of KIR-Fc fusion proteins and their use in a multiplex HLA class I binding assay. J. Immunol. Methods 425, 79-87 (2015).

229. Garcia-Diaz, A. et al. Interferon receptor signaling pathways regulating PD-L1 and PD-L2 expression. Cell Rep. 19, 1189-1201 (2017).

230. Quatrini, L. et al. Glucocorticoids and the cytokines IL-12, IL-15, and IL-18 present in the tumor microenvironment induce PD-1 expression on human natural killer cells. J. Allergy Clin. Immunol. https://doi.org/10.1016/j.jaci.2020.04.044 (2020).

231. Benson, D. M. Jr. et al. The PD-1/PD-L1 axis modulates the natural killer cell versus multiple myeloma effect: a therapeutic target for CT-011, a novel monoclonal anti-PD-1 antibody. Blood 116, 2286-2294 (2010).

232. Thomas, L. M. Current perspectives on natural killer cell education and tolerance: emerging roles for inhibitory receptors. Immunotargets Ther. 4, 45-53 (2015).

233. Shimasaki, N., Jain, A. \& Campana, D. NK cells for cancer immunotherapy. Nat. Rev. Drug Discov. 19, 200-218 (2020).

234. Benson, D. M. Jr. et al. A phase I Trial of the anti-KIR antibody IPH2101 and lenalidomide in patients with relapsed/refractory multiple myeloma. Clin. Cancer Res. 21, 4055-4061 (2015).

235. Acebes-Huerta, A. et al. Drug-induced hyperploidy stimulates an antitumor NK cell response mediated by NKG2D and DNAM-1 receptors. Oncoimmunology 5, e1074378 (2016).

236. Ferrari de Andrade, L. et al. Antibody-mediated inhibition of MICA and MICB shedding promotes NK cell-driven tumor immunity. Science 359, 1537-1542 (2018)

237. Vey, N. et al. A phase 1 study of lirilumab (antibody against killer immunoglobulin-like receptor antibody KIR2D; IPH2102) in patients with solid tumors and hematologic malignancies. Oncotarget 9, 17675-17688 (2018).

238. Lee, N. et al. HLA-E is a major ligand for the natural killer inhibitory receptor CD94/NKG2A. Proc. Natl Acad. Sci. USA 95, 5199-5204 (1998).

239. Ribas, A. \& Wolchok, J. D. Cancer immunotherapy using checkpoint blockade. Science 359, 1350-1355 (2018).

240. Lupo, K. B. \& Matosevic, S. CD155 immunoregulation as a target for natural killer cell immunotherapy in glioblastoma. J. Hematol. Oncol. 13, 76 (2020).

241. Solinas, C., Silva, De, Bron, P., Willard-Gallo, D. \& Sangiolo, K.D. Significance of TIM3 expression in cancer: from biology to the clinic. Semin. Oncol. 46, 372-379 (2019).

242. Della Chiesa, M. et al. Features of memory-like and PD-1 ${ }^{+}$human NK cell subsets. Front. Immunol. 7, 351 (2016).

243. Holubova, M. et al. Improving the clinical application of natural killer cells by modulating signals signal from target cells. Int. J. Mol. Sci. 20, 3472 (2019).

244. Li, Y., Hermanson, D. L., Moriarity, B. S. \& Kaufman, D. S. Human iPSC-derived natural killer cells engineered with chimeric antigen receptors enhance antitumor activity. Cell Stem Cell 23, 181-192 e185 (2018).

245. Szmania, S. et al. Ex vivo-expanded natural killer cells demonstrate robust proliferation in vivo in high-risk relapsed multiple myeloma patients. J. Immunother. 38, 24-36 (2015).

246. Yoon, S. R. et al. Generation of donor natural killer cells from $\mathrm{CD} 34^{+}$progenitor cells and subsequent infusion after HLA-mismatched allogeneic hematopoietic cell transplantation: a feasibility study. Bone Marrow Transplant. 45, 1038-1046 (2010)

247. Geller, M. A. et al. A phase II study of allogeneic natural killer cell therapy to treat patients with recurrent ovarian and breast cancer. Cytotherapy 13, 98-107 (2011)

248. Curti, A. et al. Successful transfer of alloreactive haploidentical KIR ligandmismatched natural killer cells after infusion in elderly high risk acute myeloid leukemia patients. Blood 118, 3273-3279 (2011).

249. Klingemann, $\mathrm{H}$. et al. Autologous stem cell transplant recipients tolerate haploidentical related-donor natural killer cell-enriched infusions. Transfusion 53, 412-418 (2013). quiz 411.

250. Shaffer, B. C. et al. Phase II study of haploidentical natural killer cell infusion for treatment of relapsed or persistent myeloid malignancies following allogeneic hematopoietic cell transplantation. Biol. Blood Marrow Transplant. 22, 705-709 (2016).

251. Lee, D. A. et al. Haploidentical natural killer cells infused before allogeneic stem cell transplantation for myeloid malignancies: a phase I trial. Biol. Blood Marrow Transplant. 22, 1290-1298 (2016).
252. Shah, N. et al. Phase I study of cord blood-derived natural killer cells combined with autologous stem cell transplantation in multiple myeloma. Br. J. Haematol. 177, 457-466 (2017).

253. Ciurea, S. O. et al. Phase 1 clinical trial using mblL21 ex vivo-expanded donorderived NK cells after haploidentical transplantation. Blood 130, 1857-1868 (2017).

254. Habib, S., Tariq, S. M. \& Tariq, M. Chimeric antigen receptor-natural killer cells: the future of cancer immunotherapy. Ochsner J. 19, 186-187 (2019).

255. Conlon, K. C. et al. Redistribution, hyperproliferation, activation of natural killer cells and CD8 T cells, and cytokine production during first-in-human clinical trial of recombinant human interleukin-15 in patients with cancer. J. Clin. Oncol. 33, 74-82 (2015).

256. Josefsson, S. E. et al. TIGIT and PD-1 mark intratumoral T cells with reduced effector function in B-cell non-Hodgkin lymphoma. Cancer Immunol. Res. 7, 355-362 (2019).

257. Becker, P. S. et al. Selection and expansion of natural killer cells for NK cell-based immunotherapy. Cancer Immunol. Immunother. 65, 477-484 (2016).

258. Burugu, S., Dancsok, A. R. \& Nielsen, T. O. Emerging targets in cancer immunotherapy. Semin. Cancer Biol. 52, 39-52 (2018).

259. Dougall, W. C., Kurtulus, S., Smyth, M. J. \& Anderson, A. C. TIGIT and CD96: new checkpoint receptor targets for cancer immunotherapy. Immunol. Rev. 276, 112-120 (2017).

260. Szturz, P. \& Vermorken, J. B. Immunotherapy in head and neck cancer: aiming at EXTREME precision. BMC Med. 15, 110 (2017).

261. Fang, L. et al. Targeting late-stage non-small cell lung cancer with a combination of DNT cellular therapy and PD-1 checkpoint blockade. J. Exp. Clin. Cancer Res. 38, 123 (2019).

262. Gao, Y. et al. Tumor immunoevasion by the conversion of effector NK cells into type 1 innate lymphoid cells. Nat. Immunol. 18, 1004-1015 (2017).

263. Suck, G. et al. NK-92: an 'off-the-shelf therapeutic' for adoptive natural killer cellbased cancer immunotherapy. Cancer Immunol. Immunother. 65, 485-492 (2016).

264. Sek, K. et al. Targeting adenosine receptor signaling in cancer immunotherapy. Int. J. Mol. Sci. 19, 3837 (2018).

265. Whiteside, T. L. Targeting adenosine in cancer immunotherapy: a review of recent progress. Expert Rev. Anticancer Ther. 17, 527-535 (2017).

266. Wang, J., Lupo, K. B., Chambers, A. M. \& Matosevic, S. Purinergic targeting enhances immunotherapy of $\mathrm{CD}^{+}{ }^{+}$solid tumors with piggyBac-engineered chimeric antigen receptor natural killer cells. J Immunother Cancer 6, 136 (2018).

267. Chambers, A. M. et al. Adenosinergic signaling alters natural killer cell functional responses. Front. Immunol. 9, 2533 (2018).

268. Torres, Á. et al. Extracellular adenosine promotes cell migration/invasion of glioblastoma stem-like cells through $\mathrm{A}_{3}$ adenosine receptor activation under hypoxia. Cancer Lett. 446, 112-122 (2019).

269. Arab, S. \& Hadjati, J. Adenosine blockage in tumor microenvironment and improvement of cancer immunotherapy. Immune Netw. 19, e23 (2019).

270. Frazao, A. et al. CD16 ${ }^{+} \mathrm{NKG} 2 \mathrm{~A}^{\text {high }}$ natural killer cells infiltrate breast cancerdraining lymph nodes. Cancer Immunol. Res. 7, 208-218 (2019).

271. Jin, D. et al. CD73 on tumor cells impairs antitumor T-cell responses: a novel mechanism of tumor-induced immune suppression. Cancer Res. 70, 2245-2255 (2010).

272. Nowak, M. et al. The A2aR adenosine receptor controls cytokine production in iNKT cells. Eur. J. Immunol. 40, 682-687 (2010).

273. Festag, J. et al. Preventing ATP degradation by ASO-mediated knockdown of CD39 and CD73 results in A2aR independent rescue of T-cell proliferation. Mol. Ther.-Nucl. Acid. 21, 656-669 (2020).

274. Horenstein, A. L., Bracci, C., Morandi, F. \& Malavasi, F. CD38 in adenosinergic pathways and metabolic re-programming in human multiple myeloma cells: in-tandem insights from basic science to therapy. Front. Immunol. 10, 760 (2019).

275. Young, A. et al. A2AR adenosine signaling suppresses natural killer cell maturation in the tumor microenvironment. Cancer Res. 78, 1003-1016 (2018).

276. Lee, M. Y. \& Allen, C. T. Mechanisms of resistance to T cell-based immunotherapy in head and neck cancer. Head Neck 42, 2722-2733 (2020).

277. Muller, L. et al. Human tumor-derived exosomes (TEX) regulate Treg functions via cell surface signaling rather than uptake mechanisms. Oncoimmunology 6, e1261243 (2017)

278. Su, W. et al. The CAMP-adenosine feedback loop maintains the suppressive function of regulatory T cells. J. Immunol. 203, 1436-1446 (2019).

279. Seitz, L. et al. Safety, tolerability, and pharmacology of AB928, a novel dual adenosine receptor antagonist, in a randomized, phase 1 study in healthy volunteers. Invest. New Drugs 37, 711-721 (2019).

280. Wang, W. T. et al. Elevated absolute NK cell counts in peripheral blood predict good prognosis in chronic lymphocytic leukemia. J. Cancer Res. Clin. Oncol. 144, 449-457 (2018). 
281. Pico de Coaña, Y., Choudhury, A. \& Kiessling, R. Checkpoint blockade for cancer therapy: revitalizing a suppressed immune system. Trends Mol. Med. 21, 482-491 (2015).

282. Willingham, S. B. et al. A2AR antagonism with $\mathrm{CPI}-444$ induces antitumor responses and augments efficacy to anti-PD-(L)1 and Anti-CTLA-4 in preclinical models. Cancer Immunol. Res. 6, 1136-1149 (2018).

283. Welihinda, A. A., Kaur, M., Raveendran, K. S. \& Amento, E. P. Enhancement of inosine-mediated $\mathrm{A}_{2 \mathrm{~A}} \mathrm{R}$ signaling through positive allosteric modulation. Cell. Signal. 42, 227-235 (2018)

284. Zhao, X., Li, L., Starr, T. K. \& Subramanian, S. Tumor location impacts immune response in mouse models of colon cancer. Oncotarget 8, 54775-54787 (2017).

285. Masjedi, A. et al. Downregulation of A2AR by siRNA loaded PEG-chitosan-lactate nanoparticles restores the $T$ cell mediated anti-tumor responses through blockage of PKA/CREB signaling pathway. Int. J. Biol. Macromol. 133, 436-445 (2019).

286. Fallah-Mehrjardi, K. et al. Pharmacological targeting of immune checkpoint A2aR improves function of anti-CD19 CAR T cells in vitro. Immunol. Lett. 223 , 44-52 (2020).

287. Yang, R. et al. Conversion of ATP to adenosine by CD39 and CD73 in multiple myeloma can be successfully targeted together with adenosine receptor A2A blockade. J. Immunother. Cancer 8, e000610 (2020).

288. Hatfield, S. M. et al. Immunological mechanisms of the antitumor effects of supplemental oxygenation. Sci. Transl. Med. 7, 277 ra230 (2015).

289. $\mathrm{Yu}, \mathrm{M}$. et al. CD73 on cancer-associated fibroblasts enhanced by the A2Bmediated feedforward circuit enforces an immune checkpoint. Nat. Commun. 11, 515 (2020).

290. Losenkova, K. et al. Compartmentalization of adenosine metabolism in cancer cells and its modulation during acute hypoxia. J. Cell Sci. 133 jcs241463 (2020).

291. Wu, M., Mei, F., Liu, W. \& Jiang, J. Comprehensive characterization of tumor infiltrating natural killer cells and clinical significance in hepatocellular carcinoma based on gene expression profiles. Biomed. Pharmacother. 121, 109637 (2020).

292. Azambuja, J. H. et al. CD73 downregulation decreases in vitro and in vivo glioblastoma growth. Mol. Neurobiol. 56, 3260-3279 (2019).

293. Allard, D., Chrobak, P., Allard, B., Messaoudi, N. \& Stagg, J. Targeting the CD73adenosine axis in immuno-oncology. Immunol. Lett. 205, 31-39 (2019).

294. Zhang, B. CD73: a novel target for cancer immunotherapy. Cancer Res. 70, 6407-6411 (2010)

295. Fujisawa, T. \& Isayama, H. Immunotherapy for pancreatic. Cancer. Juntendo Med. J. 66, 238-244 (2020).

296. Francis, D. M. \& Thomas, S. N. Progress and opportunities for enhancing the delivery and efficacy of checkpoint inhibitors for cancer immunotherapy. Adv. Drug Deliv. Rev. 114, 33-42 (2017).
297. Leone, R. D., Lo, Y. C. \& Powell, J. D. A2aR antagonists: next generation checkpoint blockade for cancer immunotherapy. Comput. Struct. Biotechnol. J. 13, 265-272 (2015).

298. Vaupel, P. \& Multhoff, G. Adenosine can thwart antitumor immune responses elicited by radiotherapy: therapeutic strategies alleviating protumor ADO activities. Strahlenther. Onkol. 192, 279-287 (2016).

299. Vigano, S. et al. Targeting adenosine in cancer immunotherapy to enhance Tcell function. Front. Immunol. 10, 925 (2019).

300. Zhu, Z. et al. Checkpoint inhibitors for stage I to III non-small cell lung cancer treated with surgery or radiotherapy with curative intent: a generic protocol. Cochrane Database Syst. Rev. 6, CD013364 (2019).

301. Nicolai, C. J. et al. NK cells mediate clearance of $C D 8^{+} \mathrm{T}$ cell-resistant tumors in response to STING agonists. Sci. Immunol. 5, eaaz2738 (2020).

302. Parihar, R. et al. NK cells expressing a chimeric activating receptor eliminate MDSCs and rescue impaired CAR-T cell activity against solid tumors. Cancer Immunol. Res. 7, 363-375 (2019).

303. Tang, M. et al. Tumoral NKG2D alters cell cycle of acute myeloid leukemic cells and reduces NK cell-mediated immune surveillance. Immunol. Res. 64, 754-764 (2016).

304. Lim, O. et al. GMP-compliant, large-scale expanded allogeneic natural killer cells have potent cytolytic activity against cancer cells in vitro and in vivo. PLOS ONE 8, e53611 (2013).

305. Han, B. et al. Altered NKp30, NKp46, NKG2D, and DNAM-1 expression on circulating NK cells is associated with tumor progression in human gastric cancer. J. Immunol. Res. 2018, 6248590 (2018).

306. Zhu, J. et al. Immunotherapy (excluding checkpoint inhibitors) for stage I to III non-small cell lung cancer treated with surgery or radiotherapy with curative intent. Cochrane Database Syst. Rev. 12, CD011300 (2017).

(i) Open Access This article is licensed under a Creative Commons Attribution 4.0 International License, which permits use, sharing, adaptation, distribution and reproduction in any medium or format, as long as you give appropriate credit to the original author(s) and the source, provide a link to the Creative Commons license, and indicate if changes were made. The images or other third party material in this article are included in the article's Creative Commons license, unless indicated otherwise in a credit line to the material. If material is not included in the article's Creative Commons license and your intended use is not permitted by statutory regulation or exceeds the permitted use, you will need to obtain permission directly from the copyright holder. To view a copy of this license, visit http://creativecommons. org/licenses/by/4.0/.

(c) The Author(s) 2020 